\title{
Preliminary Radiation Dose Assessment for the Palmerton Ore Storage Site, Palmerton, Pennsylvania
}

by M. Nimmagadda and C. Yu

Environmental Assessment and Information Sciences Division, Argonne National Laboratory, 9700 South Cass Avenue, Argonne, Illinois 60439 


\section{CONTENTS}

NOTATION $\ldots \ldots \ldots \ldots \ldots \ldots \ldots \ldots \ldots \ldots \ldots \ldots \ldots \ldots \ldots \ldots \ldots \ldots \ldots \ldots$ iv

ABSTRACT $\ldots \ldots \ldots \ldots \ldots \ldots \ldots \ldots \ldots \ldots \ldots \ldots \ldots \ldots \ldots \ldots$

1 INTRODUCTION AND HISTORY $\ldots \ldots \ldots \ldots \ldots \ldots \ldots \ldots \ldots \ldots$

2 SCENARIO DEFINITION $\ldots \ldots \ldots \ldots \ldots \ldots \ldots \ldots \ldots \ldots \ldots \ldots \ldots$

3 SOURCE TERMS $\ldots \ldots \ldots \ldots \ldots \ldots \ldots \ldots \ldots \ldots \ldots \ldots \ldots \ldots \ldots \ldots$

4 RESULTS $\ldots \ldots \ldots \ldots \ldots \ldots \ldots \ldots \ldots \ldots \ldots \ldots \ldots \ldots \ldots \ldots \ldots \ldots$

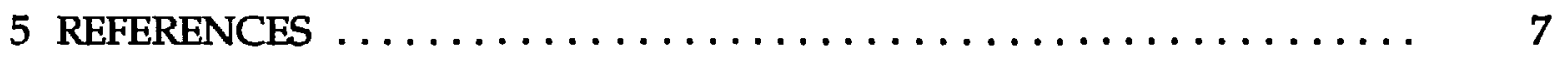

APPENDIX A: Source Term Calculation $\ldots \ldots \ldots \ldots \ldots \ldots \ldots \ldots$

APPENDIX B: Parameters Used in the Palmerton Ore Storage Site Analysis $\ldots \ldots \ldots \ldots \ldots \ldots \ldots \ldots \ldots \ldots \ldots \ldots \ldots \ldots$

APPENDIX C: RESRAD Results $\ldots \ldots \ldots \ldots \ldots \ldots \ldots \ldots \ldots \ldots \ldots \ldots$

\section{TABLES}

1 Summary of Pathways for Scenarios A, B, C, and D at POSS ........

2 Radionuclide Concentrations Used in RESRAD Computer Code for

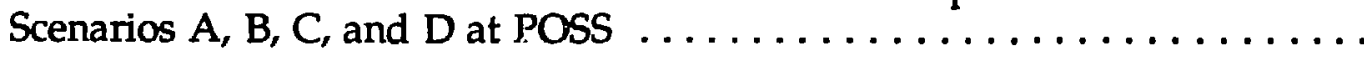

3 Summary of Potential Maximum Dose Rates for Scenarios A, B, C, and D at POSS 


\section{NOTATION}

The following is a list of the acronyms, initialisms, and abbreviations (including units of measure) used in this document.

\section{ACRONYMS, INITIALISMS, AND ABBREVIATIONS}

$\begin{array}{ll}\text { AEC } & \text { Atomic Energy Commission } \\ \text { DOE } & \text { U.S. Department of Energy } \\ \text { FUSRAP } & \text { Formerly Utilized Sites Remedial Action Program } \\ \text { ORNL } & \text { Oak Ridge National Laboratory } \\ \text { POSS } & \text { Palmerton ore storage site }\end{array}$

UNITS OF MEASURE

$\begin{array}{ll}\mathrm{cm} & \text { centimeter(s) } \\ \mathrm{cm}^{3} & \text { cubic centimeter(s) } \\ \mathrm{g} & \text { gram(s) } \\ \mathrm{in} . & \text { inch(es) } \\ \mathrm{m} & \text { meter(s) } \\ \mathrm{m}^{2} & \text { square meter(s) } \\ \mathrm{mrem} & \text { millirem(s) } \\ \mathrm{pCi} & \text { picocurie(s) } \\ \mathrm{yr} & \text { year(s) }\end{array}$




\title{
PRELIMINARY RADIATION DOSE ASSESSMENT FOR THE PALMERTON ORE STORAGE SITE, PALMERTON, PENNSYLVANIA
}

by

Manjula Nimmagadda and Charley Yu

\begin{abstract}
Potential maximum radiation dose rates were calculated for the Palmerton ore storage site in Palmerton, Pennsylvania. The RESRAD computer code, which implements the methodology described in the U.S. Department of Energy's manual for establishing residual radioactive material guidelines, was used in this evaluation. Four potential scenarios were considered for the Palmerton ore storage site. Two scenarios were developed on the basis of industrial use of the site, and two were developed on the basis of residential use of the site. The scenarios also vary with regard to time spent at the site, sources of food consumed, and source terms. The RESRAD code was used to analyze potential radiation doses from three exposure pathways. The results indicate that the basic dose limit of $100 \mathrm{mrem} / \mathrm{yr}$ would not be exceeded in Scenarios A (industrial use, hot spot), B (industrial use, homogenous contamination), and C (residential use, homogenous contamination), but would be exceeded in Scenario D (residential use, hot spot). The potential maximum dose rates for Scenarios $A, B, C$, and D are 1.0 $\mathrm{mrem} / \mathrm{yr}, 0.23 \mathrm{mrem} / \mathrm{yr}, 0.66 \mathrm{mrem} / \mathrm{yr}$, and $360 \mathrm{mrem} / \mathrm{yr}$, respectively.
\end{abstract}

\section{INTRODUCTION AND HISTORY}

The former uranium ore storage site is located in the Palmerton, Pennsylvania, area, on New Jersey Zinc Company property. Between 1953 and 1954, approximately 300 tons of ore $(0.21 \%$ uranium oxide) from the Lehigh Coal and Navigation Company were stored to support the development of eastern uranium mines and to meet the Atomic Energy Commission's (AEC's) goals for procurement and stockpiling of uranium ore. New Jersey Zinc Company accepted, sampled, and stored the ore as an agent of the AEC. The ore remained there until 1973 when, as an indirect result of the Grand Junction mill tailings legislation, the AEC initiated a program to evaluate and clean up its ore storage and stockpile locations.

The AEC cleanup plan for the Palmerton site called for the removal of the ore and the first $15 \mathrm{~cm}$ of soil. In June 1973, the ore and soil were transported to the AEC 
Feed Materials Center in Fernald, Ohio, for disposal in the plant's raffinate pits. The area at the east end of the stockpile was excavated an additional 61 to $76 \mathrm{~cm}$ to remove some chunks of ore that were buried. This material was disposed of in New Jersey Zinc Company's slag dump. The site met the guidelines adopted by the AEC at that time and was released to the owner.

As part of the Formerly Utilized Sites Remedial Action Program (FUSRAP), the U.S. Department of Energy (DOE) is implementing a radiological survey program to determine the radiological conditions at sites that were used by agencies prior to the DOE. Although the final Palmerton site report indicated that the site met the criteria as defined at the time of cleanup, the DOE determined that supporting radiological data were not sufficient to demonstrate that contemporary standards were met throughout the site. Subsequent radiological criteria and guidelines have become more stringent for the release of such sites for unrestricted use.

At the request of DOE, Oak Ridge National Laboratory (ORNL) conducted a comprehensive survey of a portion of the site in July and August of 1988. The purpose of this report is to calculate potential maximum radiation dose rates for both present and possible future use conditions. The RESRAD computer code, which implements the methodology described in DOE's manual for establishing residual radioactive material guidelines (Gilbert et al. 1989), was used to perform a dose assessment for that portion of the site surveyed by ORNL.

\section{SCENARIO DEFINITION}

Four potential exposure scenarios are considered in the dose assessment. In all scenarios, unrestricted use, at some time within 1000 years, is assumed for the Palmerton ore storage site (POSS) areas. Potential radiation doses resulting from three exposure pathways are analyzed: (1) direct exposure to external radiation from the contaminated soil material, (2) internal radiation from inhalation of contaminated dust, and (3) internal radiation from ingestion of plant foods grown in the contaminated area and irrigated with water drawn from a well adjacent to the contaminated area. On-site well water is not used for drinking because of the poor quality of shallow well water (Cottrell 1990). For Scenario D, however, it is assumed that the shallow well water is used for irrigation of garden vegetables. Also, for all scenarios, it is assumed that the worker or resident does not ingest fish, meat, or milk, from the site area.

In Scenario A (industrial use, hot spot), industrial use of the site is assumed. A hypothetical person is assumed to work in the POSS loading and unloading operations 15 minutes per week, 50 weeks per year, in the vicinity of the hot spot. Industrial use is also assumed in Scenario B (industrial use, homogeneous contamination). A hypothetical person is assumed to work in the POSS area 8 hours per day (6 hours cutdoors and 2 hours indoors), 5 days per week, 50 weeks per year. It is also assumed 
in Scenario B that the site is uniformly contaminated. For Scenarios A and B, the worker does not ingest plant foods.

Unlike Scenarios A and B, in Scenarios C (residential use, homogeneous contamination) and $D$ (residential use, hot spot), nonindustrial use of the site is assumed. In Scenarios $C$ and $D$, a hypothetical person takes up residence in the immediate vicinity of the site. The differences between Scenarios $C$ and D are the source of contamination and the consumption of plant food. In Scenario $C$, the site is assumed to be uniformly contaminated, and the resident does not consume plant food grown on-site; in Scenario $D$, the resident builds a home immediately adjacent to the hot spot and consumes plant food grown in the garden located directly over the hot spot. The resident spends 4 months per year, 2 hours per day, 6 days per week in the vicinity of the hot spot (garden).

The RESRAD computer code (Gilbert et al. 1989) was used to calculate the radiation doses to the hypothetical future resident or worker for the four scenarios, on the basis of the following assumptions:

- Scenario A

- The worker spends 15 minutes per week, 50 weeks per year, on-site, in the vicinity of the hot spot.

- The worker does not consume any meat, milk, water, aquatic food, or vegetables from the site.

- Scenario B

- The worker spends 2000 hours per year on-site.

- The walls, floor, and foundation of the industrial building reduce external exposure by 30\%; the indoor dust level is $40 \%$ of the outdoor dust level (Gilbert et al. 1989).

- The worker does not consume any plant foods, meat, milk, aquatic food, or water from the site.

- The site is covered with a 6 -in. thick crust that has a concrete-like consistency.

- Scenario C

- The resident spends $50 \%$ of his or her time indoors on the site, $25 \%$ outdoors on the site, and $25 \%$ away from the site. 
- The walls, floor, and foundation of the house reduce external exposure by $30 \%$; the indoor dust level is $40 \%$ of the outdoor dust level.

- The resident does not consume any plant foods, meat, milk, aquatic food, or water from the site.

- The site is covered with a 6 -in. thick crust that has a concrete-like consistency.

- Scenario D

- The resident spends $50 \%$ of his or her time indoors on the site, $25 \%$ outdoors on the site ( $2.3 \%$ in the vicinity of the garden), and $25 \%$ away from the site.

- The resident spends 4 months per year, 2 hours per day, 6 days per week in the vicinity of the garden.

- The walls, floor, and foundation of the house reduce external exposure by $30 \%$; the indoor dust level is $40 \%$ of the outdoor dust level.

- The external on-site outdoor radiation level away from the garden is assumed to be $80 \%$ of the radiation level in the garden. The onsite outdoor dust level is assumed to be the same as in the vicinity of the garden.

- Approximately $0.15 \%$ of the plant-food diet consumed by the resident is grown in a garden on the area of the hot spot.

- The hot spot area $\left(3 \mathrm{~m}^{2}\right)$ is not adequate to provide any meat or milk for the resident from livestock raised (i.e., foraging) on-site.

- Vegelables are irrigated with water drawn from a shallow well located adjacent to the contaminated area.

All pathways considered for Scenarios A, B, C, and D are summarized in Table 1.

\section{SOURCE TERMS}

The source terms used in the RESRAD computer code were calculated primarily on the basis of data in Cottrell, Quillen, and Crutcher (1990). For Scenarios A and D, the hot spot uranium-238 and radium-226 concentration values provided by ORNL (Cottrell 1990) were used as an upper bound estimate. In Scenarios B and C, it was assumed that 
TABLE 1 Summary of Pathways for Scenarios A, B, C, and D at POSS

\begin{tabular}{lcc}
\hline Pathway & $\begin{array}{c}\text { Scenarios A, B, } \\
\text { and C }\end{array}$ & $\begin{array}{c}\text { Scenario } \\
\text { D }\end{array}$ \\
\hline External exposure & Yes & Yes \\
Inhalation & Yes & Yes \\
Ingestion of plant foods & No & Yes \\
Ingestion of meat & No & No \\
Ingestion of milk & No & No \\
Ingestion of fish & No & No \\
Ingestion of water & No & No \\
\hline
\end{tabular}

TABLE 2 Radionuclide Concentrations Used in the RESRAD Computer Code for Scenarios A, B, C, and D at POSS

\begin{tabular}{lcc}
\hline Radionuclide & $\begin{array}{c}\text { Scenarios A and D } \\
\text { Concentration } \\
(\mathrm{pCi} / \mathrm{g})\end{array}$ & $\begin{array}{c}\text { Scenarios B and C } \\
\text { Concentration } \\
(\mathrm{pCi} / \mathrm{g})\end{array}$ \\
\hline Radium-226 & 499.2 & 2.53 \\
Uranium-238 & 499.4 & 4.2 \\
Uranium-234 & 499.4 & 4.2 \\
Uranium-235 & 23.0 & 0.19 \\
Actinium-227 & 23.0 & 0.19 \\
Protactinitim-231 & 23.0 & 0.19 \\
Lead-210 & 499.2 & 2.53 \\
Thorium-230 & 499.4 & 4.2 \\
\hline
\end{tabular}

the construction of a house or industrial building would result in excavation and mixing of soil on-site. Thus, the weighted average radionuclide concentration values for the entire site were used for Scenarios B and C. In deriving the maximum dose rates, it was assumed that uranium-238, uranium-234, and uranium-235 were present in their natural activity concentration ratio of 1:1:0.046. To obtain the concentrations of associated daughters, it was assumed that the uranium series was in secular equilibrium. The radionuclide concentrations for each scenario used in the RESRAD computer code are presented in Table 2. A detailed description of the source term calculations is provided in Appendix A. 


\section{RESULTS}

The RESRAD computer code was used to calculate the maximum dose rates for Scenarios A through $D$. The time frame considered in this analysis was 1000 years. Radioactive decay and ingrowth were considered in the calculation of the maximum dose rates. The various parameters used in the RESRAD code for this analysis are listed

in Appendix B. The calculated maximum dose rates for Scenarios A, B, C, and D are presented in Table 3. The RESRAD code summary results are presented in Appendix C.

For all scenarios, the maximum dose rates occur at time 0 (the year the radiological survey was conducted). In Scenarios A and B (industrial use) and C (residential use), the maximum dose rates would not exceed 100 mrem per year. For Scenario A (industrial use, hot spot), the maximum dose rate of 1.0 mrem per year would result from inhalation and external exposure. The maximum dose rates for Scenarios B (industrial use, homogeneous contamination) and C (residential use, homogeneous contamination) would be 0.23 and $0.66 \mathrm{mrem} / \mathrm{yr}$, respectively. For these two scenarios, the dose to the individual would result from external exposure. The potential maximum dose rates would exceed the DOE limit of $100 \mathrm{mrem} / \mathrm{yr}$ for Scenario D (residential use, hot spot). In Scenario D, the maximum dose rate of 360 $\mathrm{mrem} / \mathrm{yr}$ would result from external exposure, inhalation, and ingestion of plant foods.

TABLE 3 Summary of Potential Maximum Dose Rates for Scenarios A, B, C, and D at POSS

\begin{tabular}{|c|c|c|c|c|}
\hline \multirow[b]{2}{*}{ Pathway } & \multicolumn{2}{|c|}{ Industrial Use } & \multicolumn{2}{|c|}{ Residential Use } \\
\hline & $\begin{array}{c}\text { Scenario A } \\
\text { Hot Spot } \\
\text { (mrem/yr) }\end{array}$ & $\begin{array}{c}\text { Scenario B } \\
\text { Homogeneous } \\
\text { Contamination } \\
\text { (mrem/yr) }\end{array}$ & $\begin{array}{c}\text { Scenario C } \\
\text { Homogeneous } \\
\text { Contamination } \\
(\mathrm{mrem} / \mathrm{yr})\end{array}$ & $\begin{array}{c}\text { Scenario D } \\
\text { Hot Spot } \\
\text { (mrem/yr) }\end{array}$ \\
\hline External exposure & 0.56 & 0.23 & 0.66 & 210 \\
\hline Inhalation & 0.45 & 0 & 0 & 130 \\
\hline Ingestion of plant foods & 0 & 0 & 0 & 20 \\
\hline Ingestion of meat & 0 & 0 & 0 & 0 \\
\hline Ingestion of milk & 0 & 0 & 0 & 0 \\
\hline Ingestion of fish & 0 & 0 & 0 & 0 \\
\hline Ingestion of water & 0 & 0 & 0 & 0 \\
\hline Total & 1.0 & 0.23 & 0.66 & 360 \\
\hline
\end{tabular}

The maximum dose rate occurs at time 0 (the year the radiological survey was conducted for all scenarios). 


\section{REFERENCES}

Cottrell, W.D., 1990, "Source Term Information, Palmerton Ore Storage Site, Palmerton, Pennsylvania," letter from W.D. Cottrell (Oak Ridge National Laboratory) to C. Yu (Argonne National Laboratory), Sept. 6.

Cottrell, W.D., J.L. Quillen, and J.W. Crutcher, 1990, Results of the Radiological Survey at the Former Ore Storage Site, Palmerton, Pennsylvania (PP001), Oak Ridge National Laboratory, ORNL/TM-11218, Dec.

Gilbert, T.L., et al., 1989, A Manual for Implementing Residual Radioactive Material Guidelines, ANL/ES-160, DOE/CH/8901, prepared by Argonne National Laboratory for U.S. Department of Energy, June. 


\section{APPENDIX A:}

\section{SOURCE TERM CALCULATION}

\section{A.1 SCENARIOS A AND D}

The source terms used for Scenarios A and D in the RESRAD computer code were calculated using the data provided by Oak Ridge National Laboratory (ORNL) (Cottrell 1990). Both radium-226 and uranium-238 concentrations in the hot spot have been estimated by ORNL to be less than $500 \mathrm{pCi} / \mathrm{g}$, with the majority existing in the first foot of soil from the surface. The area of the hot spot has been conservatively estimated by ORNL to be $3 \mathrm{~m}^{2}$. Background values for radium-226 and uranium-238 were obtained from Cottrell, Quillen, and Crutcher (1990).

To obtain the concentrations of uranium-234 and uranium-235, it was assumed that uranium-238, uranium-234, and uranium- 235 were present in their natural activity concentration ratio of 1:1:0.046. The concentrations of associated daughters (actinium227, protactinium-231, lead-210, thorium-230) were obtained by assuming that the uranium series was in secular equilibrium.

\section{A.2 SCENARIOS B AND C}

The volume-weighted average radionuclide concentration values for the entire site were used to calculate the source terms for Scenarios B and C. Data were obtained from Cottrell (1990) and Cottrell, Quillen, and Crutcher (1990).

The total area of the site -- $9,662 \mathrm{~m}^{2}$ - and a depth of $1 \mathrm{~m}$ were assumed in estimating the average concentrations of radium-226 and uranium-238 for Scenarios $B$ and $\mathrm{C}$. The calculated concentrations for radium-226 and uranium-238 are $2.5 \mathrm{pCi} / \mathrm{g}$ and $4.2 \mathrm{pCi} / \mathrm{g}$, respectively. The same procedure as described for Scenarios A and D was used to calculate the concentrations of uranium-238, uranium-235, actinium-227, protactinium-231, lead-210, and thorium-230.

\section{A.3 REFERENCES}

Cottrell, W.D., 1990, "Source Term Information, Palmerton Ore Storage Site. Palmerton, Pennsylvania," letter from W.D. Cottiell (Oak Ridge National Laboratory) to C. Yu (Argonne National Laboratory), Sept. 6.

Cottrell, W.D., J.L. Quillen, and J.W. Crutcher, 1990, Results of the Radiological Survey at the Former Ore Storage Site, Palmerton, Pennsylvania (PP001), Oak Ridge National Laboratory, ORNL/TM-11218, Dec. 


\section{APPENDIX B:}

\section{PARAMETERS USED IN THE PALMERTON ORE STORAGE SITE ANALYSIS}

\section{B.1 PARAMETRIC VALUES USED IN THE RESRAD ANALYSIS}

The following parameters were used in Scenarios $A$ through $D$. The parameters that differ from the RESRAD default values are indicated by providing the scenarios and the varying values.

Area of contaminated zone:

Scenarios $A$ and $D=3.0 \mathrm{~m}^{2}$ (area of hot spot).

Scenarios $B$ and $C=9,662 \mathrm{~m}^{2}$ (total area of site).

Length parallel to aquifer flow:

Is approximately equal to the square root of the area.

Scenario $A$ and $D=1.73 \mathrm{~m}^{2}$.

Scenario $B$ and $C=98.3 \mathrm{~m}^{2}$.

Cover depth:

Scenarios B and C $=0.15 \mathrm{~m}$.

Six-inch-thick crust of concrete-like consistency.

Source: Williams 1990.

Density of cover:

Scenarios B and C $=2.25 \mathrm{~g} / \mathrm{cm}^{3}$.

Density of concrete.

Source: U.S. Department of Health, Education, and Welfare 1970.

Cover layer erosion rate:

$=0 \mathrm{~m} / \mathrm{yr}$.

Contaminated zone total porosity:

$=0.492$.

Based on silty clay soil at POSS.

Source: Gilbert et al. 1989, p. 170.

Contaminated zone effective porosity:

$=0.13$.

Average of silt and clay material, based on silty clay soil at POSS.

Source: Gilbert et al. 1989, p. 187.

Contaminated zone hydraulic conductivity:

$=32.6 \mathrm{~m} / \mathrm{yr}$.

Based on silty clay soil at POSS.

Source: Gilbert et al. 1989, p. 170. 
Contaminated zone $b$ parameter:

$=10.4$.

Based on silty clay soil at POSS.

Source: Gilbert et al. 1989, p. 170.

Precipitation:

$=0.96 \mathrm{~m} / \mathrm{yr}$.

Source: Griffin 1990.

Distance from surface to water table:

Scenarios $A$ and $D=1.52 \mathrm{~m}$; no cover material.

Scenarios B and C $=1.67 \mathrm{~m}$; cover material.

Source: Ecology and Environment, Inc. 1980.

Water table drop rate:

$=0 \mathrm{~m} / \mathrm{yr}$.

Unsaturated zone 1, thickness:

$=.52 \mathrm{~m}$.

Based on the distance from the surface to the water table.

Unsaturated zone 1, total porosity:

$=0.492$.

Based on silty clay soil at POSS.

Source: Gilbert et al. 1989, p. 170.

Unsaturated zone 1, effective porosity:

$=0.13$.

Average of silt and clay material, based on silty clay soil at POSS.

Source: Gilbert et al. 1989, p. 187.

Unsaturated zone 1, soil specific $b$ parameter:

$=10.4$.

Based on silty clay soil at POSS.

Source: Gilbert et al. 1989, p. 170.

Unsaturated zone 1, hydraulic conductivity:

$=32.6 \mathrm{~m} / \mathrm{yr}$.

Based on silty clay soil at POSS.

Source: Gilbert et al. 1989, p. 170.

Occupancy and shielding factor, external gamma:

Scenario $\mathrm{A}=0.0015$.

Scenario $B=0.21$.

Scenario $C=0.6$.

Scenario $D=0.56$.

Based on the scenario description. 
Occupancy factor, inhalation:

Scenario $\mathrm{A}=0.0015$.

Scenario $\mathrm{B}=0.19$.

Scenario $C=0.45$.

Scenario $D=0.45$.

Based on the scenario description.

Irrigation:

Scenarios A, B, and C $=0$.

Based on the scenario description (no ingestion of plant foods).

The parametric values used in the RESRAD code for the POSS analysis are provided in the following pages. All parametric values are reported to two significant figures. 
Site-Specific Paraneter Sumary

\begin{tabular}{|c|c|c|c|}
\hline Henus & Paraneter & Used & Default \\
\hline R011 & Area of contaminated zone $\left(m^{* \pm 2}\right)$ & $3.000 E+00$ & $1.000 E+04$ \\
\hline R011 & Thickness of conteminated zore $(m)$ & $3.000 \mathrm{E}-01$ & $1.000 E+00$ \\
\hline Ro11 | & Length parallel to aquifer flow (m) & $1.730 E+00$ & $1.000 E+02$ \\
\hline R011 | & Basic rediation dose limit (mrem/yr) & $1.000 E+02$ & $1.000 E+02$ \\
\hline k011 & Times for calculations $(y r)$ & $1.000 E+00$ & $1.000 E+00$ \\
\hline R011 & Tines for calculations (yr) & $1.000 E+01$ & $3.000 \mathrm{E}+00$ \\
\hline R011 & Timee for calculations (yr) & $1.000 E+02$ & $1.000 E+01$ \\
\hline RO11 & Times for calculations $(y r)$ & $2,000 E+02$ & $3.000 E+01$ \\
\hline Ro11 & Times for calculations (yr) & $4.000 E+02$ & $1.000 E+02$ \\
\hline R011 & Times for calculations $(y r)$ & $6.000 E+02$ & $3.000 E+02$ \\
\hline R011 & Times for calculations $(y r)$ & $8.000 E+02$ & $1.000 E+03$ \\
\hline R011 & Times for calculations (yr) & $1.000 E+03$ & $3.000 E+05$ \\
\hline R011 & Times for calculations ( $y r)$ & +03 & $1.000 \mathrm{E}+0.4$ \\
\hline R012 & Initial principal radionuclide $(p C i / g): A c-227$ & $00 E+01$ & $0.000 E+00$ \\
\hline R012 & Initial principal radionuclide (pci/g): $\mathrm{Pa}-231$ & $2.300 E+01$ & $0.000 E+00$ \\
\hline R012 & Initial principal radionuclide $(p i j / g): p b-210$ & $92 E+02$ & $0.000 E+00$ \\
\hline RO12 & Initial principal radionuclide $(p C i / g)$ : Ra-226 & $92 E+02$ & $0.000 E+00$ \\
\hline R012 & Initisl principal radionuclide $(p C i / j):$ Th-230 & $E+02$ & $E+00$ \\
\hline R012 & Initial principal radionuclide (pCi/g): $U-234$ & $4.994 E+02$ & $0 . \operatorname{CDOE}+00$ \\
\hline R012 & Initiai principal radionuclide (pci/g): $u-235$ & $2.300 E+01$ & $0.000 E+00$ \\
\hline R012 & Initial principal radionuclide $(p c i / g): U-238$ & & \\
\hline $\mathbf{R 0 1 3}$ & Cover depth $(\mathrm{m})$ & $\$+\infty$ & $0.000 E+00$ \\
\hline $\mathbf{R 0 1 3}$ & Density of contaninared zone $\left(9 / \mathrm{cm}^{* * 3}\right)$ & & $\$+\infty$ \\
\hline $\mathbf{R} 013$ & Contaminated zone erosion rate $(m / y r)$ & C.000E+00 & $1.000 E-03$ \\
\hline R013 & Contaminated zone total porosity & & $4.000 E-01$ \\
\hline $\mathbf{R 0 1 3}$ & Conterinated zone effoctive porosity & & \\
\hline R013 & Contaminated zone hydraulic conductivity (m/yr) & $3.260 E+01$ & $1.000 E+01$ \\
\hline R013 & Contaminated zone b paraneter & +01 & +00 \\
\hline 013 & ranspiration coeffi & & \\
\hline R013 & Precipitation $(m / y r)$ & $9.600 E-01$ & $+\infty 0$ \\
\hline R013 & Irrigation (m/yr) & -00 & 2.000E-01 \\
\hline $\mathrm{RO13}$ & Irrigation mode & over & overhead \\
\hline R013 & Runoff coefficient & $2.000 E-01$ & $2.000 E-01$ \\
\hline $\mathbf{R O 1 3}$ & Watershed area for nearby stream or pond $\left(m^{* \pm 2}\right)$ & $1.000 E+06$ & $1.000 E+06$ \\
\hline $\mathrm{R} 014$ & Density of saturated $2 \mathrm{one}\left(\mathrm{g} / \mathrm{cm}^{+*} 3\right)$ & & $1.600 E+00$ \\
\hline R014 & Saturated zone total porosity & $4.000 E-01$ & $4.000 E-01$ \\
\hline R014 & Saturated zone effective porosity & $2.000 E-01$ & $2.000 E-01$ \\
\hline R014 & Saturated zone hydraulic conductivity $(\mathrm{m} / \mathrm{yr})$ & $1.000 E+02$ & $1.000 E+02$ \\
\hline $\mathrm{R} 014$ & Saturated zone hydraulic gradient & $2.000 E-02$ & $2.000 E=02$ \\
\hline R014 & Distance from surface to water table $(\mathrm{m})$ & $1.520 E+00$ & $5.000 E+00$ \\
\hline R014 & Water table drop rate $(m / y r)$ & $0.000 E+00$ & $1.000 E-03$ \\
\hline R014 & Hell pump intake depth (m below water table) & $1.000 E+01$ & $1.000 E+01$ \\
\hline $\mathrm{R} 014$ & Model: Mondispersion (ND) or Mass-Balance (MB) & ND & i ND \\
\hline $\mathbf{R 0 1 5}$ & Number of unsaturated zone strata & 1 & 13 \\
\hline
\end{tabular}


Site-Specific Paraneter Sumary (continued)

\begin{tabular}{|c|c|c|c|}
\hline Menu I & Parcueter & Used & Default \\
\hline R015 & Unsat. zone 1, thickness (m) & $1.220 E+00$ & $4.000 E+00$ \\
\hline R015 & Unsat. zone 1 , soil density $\left(9 / \mathrm{cm}^{\star \star \hbar} 3\right)$ & $1.600 E+00$ & $1.600 E+00$ \\
\hline R015 & Unsat. zone 1, total porosity & | $\therefore .920 E-01$ & $4.000 E-01$ \\
\hline R015 & Unsat. zone 1, effective porosity & $1.300 E-01$ & $2.000 E-01$ \\
\hline R015 & Unsat. zone 1, soil-specific b parameter & $1,040 E+01$ & $5.300 E+00$ \\
\hline R015 & Unsat, zone 1, hydraulic conductivity (m/yr) & $\mid \begin{array}{l}3.260 E+01 \\
\mid\end{array}$ & $1.000 E+0 ?$ \\
\hline R016 I & Distribution coefficients for Ac-227 & $1 \quad 1$ & \\
\hline 2016 & Contaminated $20 \mathrm{me}(\mathrm{cm}+3 / \mathrm{a})$ & $|2.000 E+01|$ & $2.000 E+01$ \\
\hline R016 & Unsaturated zone $1\left(c^{2}+3 / g\right)$ & $2.000 E+01$ & $2.000 E+01$ \\
\hline R016 & Saturated 2one $\left(\mathrm{cm}^{\mathrm{m}+3 / g}\right)$ & $2.000 E+01$ & $2.000 E+01$ \\
\hline R016 & Leach rate $(/ y r)$ & $0.000 E+00$ & $0.000 E+00$ \\
\hline R016 & Distribution coefficients for Pa-231 & & \\
\hline R016 & Contaninated zone $\left(\mathrm{cm}^{\star * 3 / \mathrm{g}}\right)$ & $5.000 E+01$ & $5.000 E+01$ \\
\hline R016 & Unsaturated zone $1\left(\mathrm{~cm}^{*+3} / \mathrm{g}\right)$ & $5.000 E+01$ & $5.000 E+01$ \\
\hline R016 | & Saturated zone $\left(\mathrm{cm}^{*+3 / \mathrm{g}}\right)$ & $5.000 E+01$ & 5.000E+01 \\
\hline R016 ! & Leach rate $(/ y r)$ & $\left.\right|_{1} ^{0.000 E+00}$ & $0.000 E+00$ \\
\hline R016 & Distribution coefficients for Pb-210 & 1 & \\
\hline R016 & Contaminated $20 n e\left(\mathrm{~cm}^{+4} \mathrm{H} / \mathrm{g}\right)$ & $1.000 E+02$ & $1.000 E+02$ \\
\hline R016 & Unsaturated zone $1(\mathrm{~cm} *+3 / 8)$ & $1.000 E+02$ & $1.000 E+02$ \\
\hline R016 & Saturated zone $\left(\mathrm{cm}^{\mathrm{n}} \mathrm{H} / \mathrm{g}\right)$ & $1.000 E+02$ & $1.000 E+02$ \\
\hline R016! & Leach rate (/yr) & $0.000 E+00$ & $0.000 E+00$ \\
\hline R016 & Distribution coefficiants for Ra-226 & 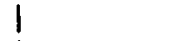 & \\
\hline R016 & Contaminated $2 / n$ e $\left(\mathrm{cm}^{\mathrm{t}}+3 / \mathrm{g}\right)$ & $7.000 E+01$ & $7.000 E+01$ \\
\hline 8016 & Unsaturafed zone $1\left(\mathrm{~cm}^{2+13 / 9}\right)$ & $7.000 E+01$ & $7.000 E+01$ \\
\hline R016 & Soturated zone $\left(\mathrm{cm}^{+*} \mathrm{H} / \mathrm{g}\right)$ & $7.000 E+01$ & $7.000 E+01$ \\
\hline R016 & Leach rate $(/ y r)$ & $0.000 E+00$ & $0.000 E+00$ \\
\hline R016 & Distribution coefficients for Th-230 & & \\
\hline R016 | & I Contaninated zone $(\mathrm{cm}+3 / \mathrm{g})$ & $6.000 E+04$ & $6.000 E+04$ \\
\hline $\mathbf{R} 016$ & Unsaturated zone $1\left(\mathrm{~cm}^{+1+3 / g}\right)$ & $6.000 E+04$ & $6.000 E+04$ \\
\hline R016 | & Saturated zone $\left(\mathrm{cm}^{\mathrm{t}}+\mathrm{3} / \mathrm{g}\right)$ & $6.000 E+04$ & $6.000 E+04$ \\
\hline 2016 | & Leach rate $(/ y r)$ & $l^{0.000 E+00}$ & $0.000 \mathrm{E}+00$ \\
\hline R016 & I Distribution coefficients for $U-234$ & & \\
\hline R016 | & Contaminated zone $(\mathrm{cm}+3 / \mathrm{g})$ & $5.000 E+01$ & $5.000 E+01$ \\
\hline R016 & Unsaturated zone $1\left(\mathrm{~cm}^{\star * 3 / g}\right)$ & $5.000 E+01$ & $5.000 E+01$ \\
\hline Ro16 i & Saturated zone $\left(\mathrm{cm}^{* * 3} / \mathrm{g}\right)$ & S.000E+01 & $5.000 E+01$ \\
\hline R016 & Leach rate $(/ y r)$ & $\begin{array}{l}0.000 E+00 \\
1\end{array}$ & $0.000 E+00$ \\
\hline R016 | & Distribution coefficients for U-235 & & \\
\hline R016 | & | Conteminated zone $\left(\mathrm{cm}^{\mathrm{m}+3 / g}\right)$ & | 5.000E+01 & $5.000 E+01$ \\
\hline R016 I & Unsaturated zone $1\left(\mathrm{~cm} \mathrm{~m}^{* * 3 / g}\right)$ & $5.000 E+01$ & $5.000 E+01$ \\
\hline $\mathbf{8 0 1 6}$ & Saturated zone $\left(\mathrm{cm}^{\mathrm{m}} \mathrm{H} / \mathrm{g}\right)$ & $5.000 E+01$ & | 5.000E+01 \\
\hline R016 | & Leach rate $(/ \mathrm{yr})$ & $0.000 E+00$ & $0.000 E+0 C$ \\
\hline
\end{tabular}


Site-specific Parameter Sumary (continued)

\begin{tabular}{|c|c|c|c|}
\hline Henu | & Paraneter & Used & Default \\
\hline R016 & Distribution coefficients for U-238 & & \\
\hline R016 & Conteminated zone $\left(\mathrm{cm}^{+* \| 3 / g}\right)$ & 5.000E+01 & $5.000 E+01$ \\
\hline R016 & Unsaturated zone $1(\mathrm{~cm} * \| 3 / \mathrm{g})$ & $5.000 E+01$ & $5.000 E+01$ \\
\hline$R 016$ & Saturated zone $(\cos +3 / g)$ & $5.000 E+01$ & $5.000 E+01$ \\
\hline R016 & Leach rate (/yr) & $0.000 E+00$ & $0.000 E+00$ \\
\hline R017 & Inhalation rate $\left(\boldsymbol{n}^{*}+3 / \mathrm{yr}\right)$ & $8.400 E+03$ & $8.400 E+03$ \\
\hline 2017 & Mass loading for inhalation $\left(9 / n^{*}+3\right)$ & $2.000 E-04$ & $2.000 E-04$ \\
\hline R017 & Dilution length for virborne dent, inhalation $(n)$ | & $3.000 E+00$ & $3.000 E \div 00$ \\
\hline$R 017$ & occupancy factor, inhalation | & $1.5 C 0 E-03$ & 4.500E-01 \\
\hline R017 & Occupency and shielding fector, external gaven & $1.500 E-03$ & $6.000 E-01$ \\
\hline $\mathbf{R} 017$ & Shepe factor, external gam & $1.000 E+00$ & $1.000 E+00$ \\
\hline R018 & Fruits, vegetables and grain consumption (kg/yr) & $0.000 E+00$ & $1.600 E+02$ \\
\hline R018 & Leafy vegetable consuption $(\mathrm{kg} / \mathrm{yr})$ & $0.000 E+00$ & $1.400 E+01$ \\
\hline R018 & Milk consuption (L/Yr) & $3.000 E+00$ & $9.200 E+01$ \\
\hline R018 & Heat and poultry consuption (kg/yr) & $0.000 E+00$ & $6.309 E+01$ \\
\hline RO18 & Fish consinption (kg/yr) & $0.000 E+00$ & $5.400 E+00$ \\
\hline R018 & ather seafood consuption (kg/yr) & $0.000 E+00$ & $9.000 E-01$ \\
\hline R018 & Orinking water intake (L/yr) & $0.000 E+00$ & $4.100 E+02$ \\
\hline $\mathbf{R 0 1 8}$ & Frection of drinking water from site & $0.000 E+00$ & $1.900 E+00$ \\
\hline R018 & Fraction of equatic food from site & $0.000 E+00$ & $5.000 E-01$ \\
\hline R019 & Livesteck fodder intake for mat (kg/day) & $6.800 E+01$ & $6.800 E+09$ \\
\hline R019 & Livestock focder Intake for milk (kg/day) & $5.500 E+01$ & S.5U0E+01 \\
\hline R019 & Livestock water intake for met (L/day) & $5.000 E+01$ & $5.000 E+01$ \\
\hline RO19 & Livestock water intake for ailk (\&/day) & $1.600 E+02$ & $1.600 \mathrm{E}+02$ \\
\hline$R 019$ & Mass laading for foliar deposition $\left(g / m^{+4} 3\right)$ & $1.000 E-04$ & $1.000 E-04$ \\
\hline$R 019$ & Depth of soll axing loyer (a) & $1.500 E-01$ & $1.500 E-01$ \\
\hline RO19 & Depth of roots $(m)$ & $9.000 E-01$ & $9.000 E-01$ \\
\hline$R 019$ & Drinking water fraction from ground water & $1.000 E+00$ & $1.000 E+00$ \\
\hline RO19 & Livestock water fraction from ground water & $1.000 E+00$ & $1.000 E+00$ \\
\hline R019 & Irrigation fraction from ground water & $1.000 E+00$ & $1.000 E+00$ \\
\hline
\end{tabular}


Site-Specific Paraneter Summary

\begin{tabular}{|c|c|c|c|}
\hline Menu 1 & Parameter & Used & Default \\
\hline Ro11 | & Area of contaminated zone $\left(m^{* \star 2}\right)$ & | $9.662 \mathrm{E}+03$ & $1.000 E+04$ \\
\hline Ro11 | & Thickness of contaminated zone (m) & $1.000 E+00$ & $1.000 E+00$ \\
\hline Ro11 | & Length parallel to aquifer flow $(\mathrm{m})$ & $9.830 E+01$ & $1.000 E+02$ \\
\hline Ro11 & Basic radiation dose limit (mrem/yr) & $1.000 E+02$ & $1.000 E+02$ \\
\hline Ro11 & Times for calculations (yr) & $1.000 E+00$ & $1.000 E+00$ \\
\hline R011 & Times for calculations (yr) & $1.000 E+01$ & $3.000 \mathrm{E}+00$ \\
\hline RO11 । & Times for calculations $(y r)$ & $1.000 E+02$ & $1.000 E+01$ \\
\hline R011 I & Times for calculations $(y r)$ & $2.000 \mathrm{E}+02$ & $3.000 \mathrm{E}+01$ \\
\hline Ro11 & Times for calculations (yr) & $4.000 E+02$ & $1.000 E+02$ \\
\hline RO11 | & Times for calculations $(y r)$ & $6.000 E+02$ & $3.000 E+02$ \\
\hline R011 ! & Times for calculations (yr) & $8.000 E+02$ & $1.000 E+03$ \\
\hline ROII | & $T$ imes for calculations (yr) & $1.000 E+03$ & $3.000 E+03$ \\
\hline R011! & Times for calculations (yr) & $2.000 E+03$ & $1.000 E+04$ \\
\hline RO12 | & Initial principal radionuclide (pCi/g): $\quad$ Ac- 227 & $1.900 E-01$ & $0.000 E+00$ \\
\hline R012 | & Initial principal radionuclide (pci/g): $\mathrm{Pa}-231$ & $1.900 E-01$ & $0.000 E+00$ \\
\hline R012 | & Initial principal radionuclide (pci/g): $\mathrm{Pb}-210$ & $2.530 E+00$ & $0.000 E+00$ \\
\hline R012 & Initial principal radionuclide $(p c i / 9)$ : $2 a-226$ & $2.530 E+00$ & $0.000 E+00$ \\
\hline R012 & Initial principal radionuclide $(p c i / g)$ : Th-230 & $4.200 E+00$ & $0.000 E+00$ \\
\hline R01? | & Initial principal radionuelide (pci/g): U-234 & $4.200 E+00$ & $0.000 E+00$ \\
\hline R012 & Initial principal radionuclide $(p c i / g): ~ U-235$ & $1.900 E-01$ & $0.000 \mathrm{E}+00$ \\
\hline R012 & Initial principal radionuclide (pci/g): U-23B & $4.200 E+00$ & $0.000 E+00$ \\
\hline R013 & Cover depth $(m)$ & $1.500 E-01$ & $0.000 E+00$ \\
\hline $\mathrm{RO} 13$ & Density of cover material $\left(\mathrm{g} / \mathrm{cm}^{+* 3}\right)$ & $2.250 E+00$ & $1.600 E+00$ \\
\hline R013 & Cover depth erosion rate $(m / y r)$ & $0.000 E+00$ & $1.000 \mathrm{E}-03$ \\
\hline R013 & Density of contaminated zone $\left(\mathrm{g} / \mathrm{cm}^{* * 3}\right)$ & $1.600 E+00$ & $1.600 E+00$ \\
\hline R013 & Contaminated zone erosion rate $(\mathrm{m} / \mathrm{yr})$ & $E+00$ & $\mathrm{IE}-03$ \\
\hline R013 & Contaminated zone total porosity & $4.920 E-01$ & $4.000 E-01$ \\
\hline RO13 & Contaminated zone effective porosity & $1.30 G E-01$ & $2.000 E-01$ \\
\hline R013 & Contaminated zone hydraulic conductivity $(m / y r)$ & $3.260 E+01$ & \\
\hline R013 & Contaminated zone b parameter & $1.040 E+01$ & $5.300 E+00$ \\
\hline $\mathrm{R} 013$ & Evapotranspiration coefficient & $6.000 E-01$ & $6.000 E-0 !$ \\
\hline R013 & Precipitation $(m / y r)$ & $\mathrm{E}-01$ & $1.000 E+00$ \\
\hline R013 & Irrigation (m/yr) & $0.000 E+00$ & $2.000 E-01$ \\
\hline R013 & irrigarion mode & overhead & overhead \\
\hline R013 & Runoff coefficient & $2.000 E-01$ & $2.000 E-01$ \\
\hline R013 & Watershed area for nearby strean or pand $\left(m^{*+2}\right)$ & $1.000 E+06$ & $1.000 E+06$ \\
\hline R014 & Density of saturated 2 one $\left(\mathrm{g} / \mathrm{cm}^{*+3}\right)$ & $1.600 E+00$ & $1.600 E+00$ \\
\hline R014 & Saturated zone total porosity & $4.000 E-01$ & 4.000E-01 \\
\hline R014 & Saturated zone effective porosity & $2.000 E-01$ & $2.000 E-01$ \\
\hline R014 & Saturated zone hydraulic conductivity $(\mathrm{m} / \mathrm{yr})$ & $1.000 E+02$ & $1.000 E+02$ \\
\hline R014 & Saturated zone hydraulic gradient & $2.000 E-02$ & $2.000 \mathrm{E}-02$ \\
\hline R014 & Distance from surface to water table $(m)$ & $1.670 E+00$ & $5.000 E+00$ \\
\hline R014 & Water table drop rate $(m / y r)$ & $0.000 E+00$ & $1.000 E \cdot 03$ \\
\hline R014 & Well pump intake depth (m below water table) & $1.000 \mathrm{E}+01$ & $1.000 E+01$ \\
\hline R014 & Model: Nondispersion (ND) or Mass-Balance (MB) & MD & ND \\
\hline R015 & Number of unsaturated zone strata & 1 & 1 \\
\hline
\end{tabular}


site-Specific Parameter Sumary (continued)

\begin{tabular}{|c|c|c|c|}
\hline Menu 1 & Parameter & Used & Default \\
\hline R015 & Uncat. zone 1, thickness $(m)$ & | 5.200E-01 & $4.000 E+00$ \\
\hline RO15 । & Unsat. zone 1 , soil density $\left(\mathrm{g} / \mathrm{cm}^{* \pm} 3\right)$ & $1.600 E+00$ & $1.600 E+00$ \\
\hline R015 । & Unsat. zone 1, total porosity & $|4.920 E-01|$ & $4.000 \mathrm{E}-01$ \\
\hline R015 । & Unsat. zone 1, effective porosity & $|1.300 E-01|$ & 2.00nE-01 \\
\hline R015 | & Unsat. zone 1, soil-specific b parameter & $|1.040 E+01|$ & $5.300 E+00$ \\
\hline R015 ! & Unsat. zone 1, hydraulic conductivity $(m / y r)$ & $3.260 E+01 \mid$ & $1.000 E+02$ \\
\hline & & 1 & \\
\hline R016 I & Distribution coefficients for Ac-227 & 1 & \\
\hline R016 | & Conteminated zone $\left(\mathrm{cm}^{* 4} 3 / \mathrm{g}\right)$ & $2.000 E+01$ & $2.000 E+01$ \\
\hline R016 | & Unsaturated zone $1\left(\mathrm{~cm}^{+* 3 / g}\right)$ & | 2.000E+01 & $2.000 E+01$ \\
\hline$R 016$ & Saturated zone $\left(\mathrm{cm}^{* * 3 / g}\right)$ & 2.000E+01 & $2.000 E+01$ \\
\hline R016 & Lnach rate $(/ y r)$ & $\left\{\begin{array}{l}0.000 E+00 \\
\end{array}\right.$ & $\mid 0.000 E+00$ \\
\hline R016 & Distribution coefficients for Pa-231 & 1 & \\
\hline R016 & Contaminated zone $\left(\mathrm{cm}^{* * 43 / g)}\right.$ & | $5.000 E+01$ & $5.000 E+01$ \\
\hline R016 & Unsaturated zone $1(c+* * 3 / g)$ & $5.000 E+01$ & $5.000 k+01$ \\
\hline R016 & Ssturated zpre $(\mathrm{cm}+3 / g)$ & $5.000 E+01$ & $5.000 E+01$ \\
\hline R016 & Leach rate $(/ y r)$ & $\left.\right|^{0.000 E+00}$ & $i^{0.000 E+00}$ \\
\hline R016 & | Distribution coefficients for $\mathrm{Pb}-210$ & 1 & 1 \\
\hline R016 & Contaminsted zone $\left(\mathrm{cm}^{* * 3 / g}\right)$ & $1.000 E+02$ & $1.000 E+02$ \\
\hline R016 & Unsaturated zone $1\left(\mathrm{~cm}^{*}+3 / \mathrm{g}\right)$ & $1.000 E+02$ & $1.000 E+02$ \\
\hline R016 & Saturated Isene $(\mathrm{cm}+3 / \mathrm{g})$ & $1.000 E+02$ & $1.000 \mathrm{E}+02$ \\
\hline R016 & Leach rate $(/ y r)$ & \}$^{0.000 E+00}$ & $\left\{\begin{array}{l}0.000 E+00 \\
1\end{array}\right.$ \\
\hline R016 & | Distribution coefficients for Ra-226 & 1 & 1 \\
\hline R016 & | Contaminated zone $\left(\mathrm{cm}^{*} * 3 / \mathrm{g}\right)$ & $7.000 E+01$ & $7.000 E+01$ \\
\hline R016 & Unsaturated zone $1(\mathrm{~cm}+\mathrm{m} / \mathrm{g})$ & | 7.000E+01 & 1 7.000E+01 \\
\hline R016 & Saturated zone $\left(\mathrm{cm}^{*} * 3 / g\right)$ & | $7.000 E+01$ & $7.000 E+01$ \\
\hline R016 & Leach rate $(/ y r)$ & $0.000 E+00$ & $0.000 E+00$ \\
\hline R016 & | Distribution coefficients for Th-230 & 1 & 1 \\
\hline 8016 & | Contaminated zone $\left(\mathrm{cm}^{*} * 3 / \mathrm{g}\right)$ & $6.000 E+04$ & $6.000 E+04$ \\
\hline R016 & Unsaturated zone $1(\mathrm{~cm}+3 / g)$ & $6.000 E+04$ & $6.000 E+04$ \\
\hline R016 & Saturated zone $(\mathrm{cm} * 3 / \mathrm{g})$ & $16.000 E+04$ & $6.000 E+04$ \\
\hline R016 & Leach rate $(/ y r)$ & $0.000 E+00$ & $\left\{\begin{array}{l}0.000 E+00 \\
\end{array}\right.$ \\
\hline R016 & I Distribution coefficients for U-234 & 1 & I \\
\hline R016 & I Contaminated zone $\left(\mathrm{Cm}^{n}+3 / g\right)$ & 1 $5.000 E+01$ & | $5.000 E+01$ \\
\hline R016 & Unsaturated zone $1(\mathrm{~cm} * 3 / \mathrm{g})$ & | $5.000 E+01$ & 5.000E $\div 01$ \\
\hline R016 & Saturated zone $\left(\mathrm{Cn}^{* 1} 3 / \mathrm{g}\right)$ & | $5.000 E+01$ & | $5.000 E+01$ \\
\hline R016 & Leach rate $(/ y r)$ & $0.000 E+00$ & $0.000 E+00$ \\
\hline & 1 & 1 & 1 \\
\hline R016 & Distribution coefficients for U- 235 & 1 & 1 \\
\hline R016 & Contaminated zone $\left(\mathrm{cm}^{*} \mathrm{~m} / \mathrm{g}\right)$ & | $5.000 E+01$ & $5.000 E+01$ \\
\hline R016 & Unsaturated zonc $1\left(\mathrm{~cm}^{*}+3 / \mathrm{g}\right)$ & | $5.000 E+01$ & $5.000 E+01$ \\
\hline R016 & Saturated zone $\left(\mathrm{Cm}^{2+3 / 3}\right)$ & $5.000 E+01$ & | $5.000 E+01$ \\
\hline RU10 & Leach rate (/yr) & $0.000 E+00$ & $0.000 E+00$ \\
\hline
\end{tabular}


Site-Specific Parameter Sumary (cont inued)

\begin{tabular}{|c|c|c|c|}
\hline Menu I & Parameter & Used & Default \\
\hline R016 & Distribution coefficients for $U-238$ & & \\
\hline RU16 । & Contaninated zone $\left(\mathrm{cm}^{*}+3 / \mathrm{g}\right)$ & $5.000 E+01$ & $5.000 E+01$ \\
\hline R016 i & Unsaturated zone $1(\mathrm{~cm}+3 / 9)$ & $5.000 E+01$ & $5.000 E+01$ \\
\hline R016 1 & Saturated zone $\left(\mathrm{cm}^{+4}+3 / g\right)$ & $5.000 E+01$ & $5.000 E+01$ \\
\hline R016 । & Leach rate $(/ y r)$ & $0.000 E+00$ & $0.000 E+00$ \\
\hline R017 & Inhalation rote $\left(m^{* *} 3 / y r\right)$ & $8.400 E+03$ & | $8.400 E+03$ \\
\hline R017 & Mass loading for inhalation $\left(9 / m^{\star \star * 3}\right)$ & $2.000 \mathrm{E}-04$ & $2.000 E-04$ \\
\hline R017 & Dilution length for airborne dust, irialation (m)| & $3.000 E+00$ & $3.000 E+00$ \\
\hline R017 & occupancy factor, inhalation | & $1.900 E-01$ & $4.500 E-01$ \\
\hline R017 । & Dccupancy and shielding factor, external gama & $2.100 E-01$ & $6.000 E-01$ \\
\hline R017 & Shape factor, external gamma & $1.000 E+00$ & $1.000 E+00$ \\
\hline R018 & Fruits, vegetables and grain consumption $(\mathrm{kg} / \mathrm{yr})$ & $0.000 E+00$ & $1.600 E+02$ \\
\hline R018 & Leafy vegetable consumption (kg/yr) & $0.000 E+00$ & $1.400 E+01$ \\
\hline R018 & Milk consumption $(L / y r)$ & $0.000 E+00$ & $9.200 E+01$ \\
\hline R018 & Meat and poultry consumption (kg/yr) & $0.000 E \sim 00$ & $6.300 E+01$ \\
\hline R018 & Fish consumption (kg/yr) & $0.000 E+00$ & $5.400 \varepsilon+00$ \\
\hline R018 & other seafood consumption (kg/yr) & $0.000 E+00$ & $9.000 \mathrm{E}-01$ \\
\hline R018 & Drinking water intake (L/Yr) & $0.000 E+00$ & $4.100 E+02$ \\
\hline R018 & Fraction of drinking water from site & $0.000 E+00$ & $1.000 E+00$ \\
\hline R018 & fraction of rquatic food from site & $0.000 E+00$ & 5.000E-01 \\
\hline R019 & Livestock fodder intake for meat ( $\mathrm{kg} /$ day) & $6.800 E+01$ & $6.800 E+01$ \\
\hline R019 & Livestock fodder intake for milk (kg/day) & $5.500 E+01$ & $5.500 E+01$ \\
\hline ROI9 & Livestock water intake for meat (L/day) & $5.000 E+01$ & $5.000 E+01$ \\
\hline R019 & Livestock water intake for milk (L/day) & $1.600 E+02$ & $1.600 E+02$ \\
\hline R019 & Mass loading for foliar deposition $\left(g / m^{* \hbar 3}\right)$ & $1.000 E-04$ & $1.000 E-04$ \\
\hline R019 & Depth of soil mixing layer $(m)$ & $1.500 E-01$ & $1.500 E-01$ \\
\hline R019 & Depth of roots $(m)$ & 9.020E-01 & $9.000 E-01$ \\
\hline R019 & Drinking water fraction from ground water & $1.000 E+00$ & $1.000 E+00$ \\
\hline R019 & Livestock water fraction from ground water & $1.000 E+00$ & | $1.000 E+00$ \\
\hline Ro19 & Irrigation fraction from ground water & $1.000 E+00$ & $1.000 \mathrm{E}+00$ \\
\hline
\end{tabular}


Site-Specific Parameter Sumary

\begin{tabular}{|c|c|c|c|}
\hline Menu | & Paraneter & Used & Default \\
\hline 2011 & Area of contaminated zone $\left(m^{\text {t*t}} 2\right)$ & $9.662 E+03$ & $1.090 E+04$ \\
\hline R011 & Thickness of contaminated zone (m) & $1.000 E+00$ & $1.000 E+00$ \\
\hline R011 & Length parallel to aquifer flow (m) & $9.830 E+01$ & $1.000 E+02$ \\
\hline R011 & Basic radiation dose limit (mrem/yr) & $1.000 E+02$ & $1.000 E+02$ \\
\hline RO11 & Times for calculations (yr) & $1.000 E+00$ & $1.000 E+00$ \\
\hline R011 & Times for calculations (yr) & $1.000 E+01$ & $3.000 E+00$ \\
\hline R011 & Tines for calculations ( $y r)$ & $1.000 E+02$ & $1.000 E+01$ \\
\hline R011 & Times for calculations $(y r)$ & $2.000 E+02$ & $3.000 E+01$ \\
\hline R011 & Times for calculations (yr) & $4.000 E+02$ & $1.000 E+02$ \\
\hline $\mathbf{R 0 1 1}$ & iimes for calculations (yr) & $6.000 E+02$ & $3.000 E+122$ \\
\hline R011 & Times for calculations (yr) & $8.000 E+02$ & $1.000 E+0 S$ \\
\hline R011 & Times for calculations $(y r)$ & $1.000 F+03$ & $3.000 E+03$ \\
\hline R011 & Times for calculations (yr) & $2.000 E+03$ & $1.000 E+04$ \\
\hline R012 & Initial principal radionkelide $(\mathrm{pCi} / g)$ : $A c-227$ & $1.900 E-01$ & $0.000 E+00$ \\
\hline R012 & Initial principal radionuclide (pci/g): $P_{a-231}$ & $1.900 E-01$ & $0.000 E+00$ \\
\hline R012 & Initial principal radionuclide $(p \mathrm{C} i / g)$ : $\mathrm{Pb}-210$ & $2.530 \mathrm{E}+00$ & $0.000 E+00$ \\
\hline R012 & Initial principal radionuclide $(p C i / g):$ Ra-226 & $2.530 E+00$ & $0.000 E+00$ \\
\hline R012 & Initial principal radionuclide (pCi/g): Th-230 & $4.200 E+00$ & $0.000 E+00$ \\
\hline R012 & Initial principal radionuclide (pci/g): U-234 & $4.200 E+00$ & $0.000 E+00$ \\
\hline R012 & Initial principal radionuclide (pci/g): $u-235$ & $1.900 E-01$ & $0.000 E+00$ \\
\hline R012 & Initial principal radionuclide (pCi/3): U-238 & $4.200 E+00$ & $0.000 E+00$ \\
\hline R013 & Cover depth (m) & $1.500 E-01$ & $0.000 E+00$ \\
\hline R013 & Density of cover material $\left(g / \mathrm{cm}^{* * 3}\right)$ & $2.250 E+00$ & $1.600 E+00$ \\
\hline R013 & Cover depth erosion rate ( $m / y r)$ & $0.000 E+00$ & $1.000 E-03$ \\
\hline R013 & Density of conteminated $z$ one $\left(9 / \mathrm{cm}^{\star \star} * 3\right)$ & $1.600 E+00$ & $1.600 E+00$ \\
\hline R013 & Contaminated zone erosion rate (m/yr) & $E+00$ & $E-03$ \\
\hline $\mathbf{k 0 1 3}$ & Contaminated zone total porosity & $4.920 E-01$ & $4.000 E-01$ \\
\hline R013 & Contaninated zone effective porosity & $1.300 E-01$ & 2.000E-01 \\
\hline R013 & Contaminated zone hydraulic conductivity $(m / y r)$ & $3.260 E+01$ & $1.000 E+01$ \\
\hline R013 & Contaminated zone b paraneter & $1.040 E+01$ & $5.300 E+00$ \\
\hline R013 & Evapotranspiratien coefficient & $6.000 E-01$ & $6.000 E-01$ \\
\hline R013 & Precipitation (m/r) & $9.600 E-01$ & $1.000 E+00$ \\
\hline R013 & Irrigation $(m / y r)$ & $0.000 E+00$ & $2.000 E-01$ \\
\hline R013 & Irrigation mode & | overhead & overhead \\
\hline R013 & Runoff coef $t$ icient & $2.000 E-01$ & $2.000 E-01$ \\
\hline R013 & Watershed area for nearby stream or pond $\left(m^{* \star 2}\right)$ & $1.000 E+06$ & $1.000 E+06$ \\
\hline R014 & Density of saturatzd zone $\left(g / \mathrm{cm}^{ \pm * 3}\right)$ & $1.600 E+00$ & $1.600 E+00$ \\
\hline R014 & Saturated zone total porosity & $4.000 E-01$ & $4.000 E-01$ \\
\hline R014 & Saturated zone effective porosity & | $2.000 E-01$ & $2.000 \mathrm{E}-01$ \\
\hline R014 & Saturated zone hydraulic conductivity (m/yr) & | $1.000 E+02$ & $1.000 E+02$ \\
\hline R014 & Saturated zone hydraulic gradient & $2.000 E-02$ & $2.000 E \cdot 02$ \\
\hline R014 & Distance from surface to water table $(\mathrm{m})$ & | $1.670 E+00$ & $5.000 E+00$ \\
\hline R014 & Water table drop rate $(\mathrm{m} / \mathrm{yr})$ & $0.000 E+00$ & $1.000 E-03$ \\
\hline R014 & Well pump intake depth (m below water table) & $1.000 \mathrm{E}+01$ & | $1.000 E+01$ \\
\hline $\mathbf{R 0 1 4}$ & Model: Hondispersion (ND) or Mass-Balance (MB) & I ND & No \\
\hline R015 & Number of unsaturated zone strata & 11 & 1 \\
\hline
\end{tabular}


Site-Specific Parameter Sumary (cont inued)

\begin{tabular}{|c|c|c|c|}
\hline Henu 1 & Parameter & Used & Default \\
\hline R015 & Unsat. zone 1, thickness (m) & $5.200 E-01$ & $4.000 E+00$ \\
\hline R015 & Unsat. zone 1 , soil density $\left(\mathrm{g} / \mathrm{cm}^{\star \star * 3}\right)$ & $1.600 E+00$ & $1.600 E+00$ \\
\hline R015 & Unsat. zone 1, total porosity & $4.920 E-01$ & $4.000 E-01$ \\
\hline R015 & Unsat. zone 1, effective porosity & $1.300 E-01$ & $2.000 E-01$ \\
\hline R015 & Unsat. zone 1 , soil-specific b parameter & $1.040 E+01$ & $5.300 E+00$ \\
\hline RO15 & Unsat. zone 1 , hydraul ic conduct ivity $(m / y r)$ & $3.260 E+01$ & $1.000 E+02$ \\
\hline R016 | & Distribution coefficients for AC- 227 & & \\
\hline R016 । & Contaminated zone $(\mathrm{cm} * 3 / g)$ & $2.000 E+01$ & $2.000 E+01$ \\
\hline R016 I & Unsaturated zone $1\left(\mathrm{~cm}^{* * 3 / g}\right)$ & $2.000 E+01$ & $2.000 E+01$ \\
\hline R016 i & Saturated zone $(\mathrm{cm} * * 3 / \mathrm{g})$ & $2.000 E+01 !$ & ! 2.50it:01 \\
\hline R016 | & Leach rate $(/ y r)$ & i $0.005=015$ & $0.000 E+00$ \\
\hline R016 | & Distribution coefficients $f a t$ ie- & & \\
\hline R016 & Contaninated zone $(\mathrm{cm}=3 / \mathrm{g})$ & $5.000 E+01 \mid$ & 5.000E+01 \\
\hline R016 | & Unsaturated zone $1\left(\mathrm{~cm}^{*+3 / g}\right)$ & j.000E+01 & $5.000 E+01$ \\
\hline 2016 & Saturated zone $\left(\mathrm{cm}^{\star n} 3 / \mathrm{g}\right)$ & $5.000 E+01$ & $5.000 E+01$ \\
\hline R016 I & Leach rate $(/ y r)$ & $0.000 E+00$ & $0.000 E+00$ \\
\hline R016 I & Distribution coefficients for Pb-210 & & \\
\hline R016 I & Contaminated zone $(\mathrm{cm} * * 3 / \mathrm{g})$ & $1.000 E+02$ & $1.000 E+62$ \\
\hline R016 | & Unsaturated zone $1(\mathrm{~cm} * 3 / 3)$ & $1.000 E+02$ & $1.000 E+02$ \\
\hline R016 | & Saturated zone $\left(\mathrm{cm}^{*}+3 / \mathrm{g}\right)$ & $1.000 E+02$ & $1.000 E+02$ \\
\hline R016 | & Leach rate $(/ y r)$ & $0.000 E+00$ & $0.000 E+00$ \\
\hline R016 | & Distribution coefficients for Ra-226 & & \\
\hline R016 | & Contaninated zone $(\mathrm{cm} * 3 / \mathrm{g})$ & $7.000 E+01$ & $7.000 E+01$ \\
\hline R016 i & Unsaturated zone $1\left(\mathrm{~cm}^{* * 3 / g}\right)$ & | 7.000E+01 & $7.000 E+01$ \\
\hline en1s i & Saturatad zone $\left(\mathrm{cm}^{* * 3 / g}\right)$ & $7.000 E+01$ & $7.000 E+01$ \\
\hline R016 & Leach rate $(/ y r)$ & $10.000 E+00$ & $0.000 E+00$ \\
\hline R016 | & Distribution coefficients for Th-230 & & \\
\hline R016 | & Contaminated zone $\left(\mathrm{cm}^{ \pm \star 3 / g}\right)$ & $6.000 E+04$ & $6.000 E+04$ \\
\hline R016 I & Unsaturated zone $1(\mathrm{~cm} * 3 / \mathrm{g})$ & $6.000 E+04$ & $6.000 E+04$ \\
\hline R016 I & Saturated zone $\left(\mathrm{cm}^{\star \star} 3 / \mathrm{g}\right)$ & $6.000 E+04$ & $6.000 E+04$ \\
\hline R016 | & Leach rate $(/ y r)$ & $0.000 E+00$ & $0.000 E+00$ \\
\hline R016 | & Distribution coefficients for U-234 & & \\
\hline R016 | & Contaminated zone $\left(\mathrm{cm}^{\mathrm{t}+3 / \mathrm{g}}\right)$ & $5.000 E+01$ & | $5.000 E+01$ \\
\hline R016 | & Unsaturated zone $q(\mathrm{~cm} * \| 3 / \mathrm{g})$ & $5.000 E+01$ & $5.000 E+01$ \\
\hline R016 | & Saturated zone $(\mathrm{cm} * * 3 / \mathrm{g})$ & $5.000 E+01$ & $5.000 E+0 i$ \\
\hline R016 & Leach rate $(/ y r)$ & $0.0 n 0 E+00$ & $0.000 E+00$ \\
\hline R016 & Distribution coefficients for U-235 & & \\
\hline R016 & | Contaninated zone $(\mathrm{cm} * * 3 / g)$ & $5.000 E+01$ & $5.000 E+01$ \\
\hline R016 & Unsaturated zone $1\left(\mathrm{~cm}^{*+3 / g}\right)$ & $5.000 E+01$ & $5.000 E+01$ \\
\hline R016 & Saturated zone $(\mathrm{cm} * * 3 / g)$ & $5.000 E+01$ & $5.000 E+01$ \\
\hline R016 & Leach rate $(/ Y \Gamma)$ & $0.000 E+00$ & $10.000 E+00$ \\
\hline
\end{tabular}


site-Specific Parmeter Sumary (continued)

\begin{tabular}{|c|c|c|c|}
\hline Meras | & Parameter & Used & Default \\
\hline R016 | & Distribution eoeffieients for $U-238$ & & \\
\hline R016 i & Contaninated zone $\left(\operatorname{con}^{+13} 3 / g\right)$ & $5.000 E+01$ & $5.000 E+01$ \\
\hline R016 | & Unsaturated zone i (cat+3/g) & $5.000 E+09$ & $5.000 E+01$ \\
\hline R016 | & Saturated zone $(\mathrm{cm}+\mathrm{m} / \mathrm{g})$ & $5.000 E+01$ & $5.000 E+01$ \\
\hline RO16 i & Leach rate $(/ y r)$ & $0.000 E+00$ & $0.000 \mathrm{E}+00$ \\
\hline R017 & Inhalation rate $(\mathbf{m + 3 / y r )}$ & $8.400 E+03$ & $8.400 E+03$ \\
\hline R017 & Mass looding for inhalation $\left(g / m^{*+3}\right)$ & $2.000 E-04$ & $2.000 E-0.4$ \\
\hline R017 & Dilution length for airborne dust, inhalation (m)) & $3.000 E+00$ & $3.000 E+00$ \\
\hline R017 & Occupency factor, inhalation & $4.500 E-01$ & $4.500 E-01$ \\
\hline R017 & Decupancy and shielding factor, external gamma & $6.000 E-01$ & $6.000 E-01$ \\
\hline R017 & Shape factor, external gama & $1.000 E+00$ & $1.000 E+00$ \\
\hline RO18 | & | Fruits, vegetables and srain consumption (kg/yr) | & $0.000 E+00$ & $1.600 E+02$ \\
\hline R018 | & | Leafy vegetable consuption (kg/yr) & $0.000 E+00$ & $1.400 E+01$ \\
\hline ROT8 | & I Milk ensuption (L/Yr) & $0.000 E+00$ & $9.200 E+01$ \\
\hline R018 & Neat and poultry consuption (kg/yr) & $0.000 E+00$ & $6.300 E+01$ \\
\hline R018 & Fish consuption (kg/vr) & $0.000 E+00$ & $5.400 E+00$ \\
\hline RU18 & Other seafood consureption (kg/yr) & $0.000 E+00$ & $9.000 E-01$ \\
\hline R018 & Orinking water intake $(L / Y r)$ & $0.000 E+00$ & $4.100 E+02$ \\
\hline R018 & Fraction of drinking water from site & $0.000 E+00$ & $1.000 \mathrm{E}+00$ \\
\hline R018 & Froction of equatic food frow site & $0.000 E+00$ & $5.000 E-01$ \\
\hline R019 & Livestock fodder intake for weat (kg/day) & | $6.800 E+01$ & $6.800 E+01$ \\
\hline R019 & Livestock fodder intake for milk (kg/day) & | 5.500E+01 & $5.500 E+01$ \\
\hline R019 & | Livestock water intake for neat (L/day) & $5.000 E+01$ & $5.000 E+01$ \\
\hline 2019 & I Livestock water intake for ailk (L/day) & $1.600 E+02$ & $1.600 E+02$ \\
\hline R019 ! & I Mase laading for foliar deposition $\left(g / \mathrm{m}^{-m} 3\right)$ & 1.000 -04 & $1.000 E-04$ \\
\hline R019 & Depth of soll wixing leyer (m) & | 1.500E-01 & $1.500 E-01$ \\
\hline$R 019$ & Depth of roots (m) & $9.000 E-01$ & $9.000 E-01$ \\
\hline R019 & Drinking water fraction from ground water & $1.000 E+00$ & $1.000 E+00$ \\
\hline R019 & Livestock water fraction from ground water & | $1.000 E+00$ & | $1.000 \mathrm{E}+00$ \\
\hline R019 & Irrigation fraction from ground water & $1.000 E+00$ & $11.000 E+00$ \\
\hline
\end{tabular}


Site-Specific Paraneter Sumary

\begin{tabular}{|c|c|c|c|}
\hline Menu $\mid$ & Parameter & Used & Default \\
\hline R011 & Area of costaminated zone $\left(\mathrm{A}^{+\star} 2\right)$ & $3.000 E+00$ & $1.000 E+06$ \\
\hline R011 & Thickness of contaminated zone $(m)$ & $3.000 \mathrm{E}-01$ & $1.000 E+00$ \\
\hline R011 & Length parall $=l$ to aquifer flow (m) & $1.730 E+00$ & $1.000 E+02$ \\
\hline R011 & Easic radiation dose limit (mren/yr) & $1.000 E+02$ & $1.000 E+02$ \\
\hline R011 I & Times for calculations $(y r)$ & $1.000 E+00$ & $1.000 E+00$ \\
\hline R011 | & Times for calculations (yr) & $1.000 E+01$ & $3.000 E+00$ \\
\hline R011 & Times for calculations $(y r)$ & $1.000 E+02$ & $1.000 \mathrm{E}+01$ \\
\hline R011 & Times for calculations (yr) & | $2.000 E+02$ & $3.000 E+01$ \\
\hline R011 & Times for calculations (yr) & $4.000 E+02$ & $1.000 E+02$ \\
\hline R011 & Times for calculations (yr) & $6.000 E+02$ & $3.000 E+02$ \\
\hline 2511 & Times for calculations $(y r)$ & $8.000 E+02$ & $1.000 E+03$ \\
\hline R011 & Times for calculations (yr) & $1.000 E+03$ & $3.000 E+03$ \\
\hline R011 & rimes for calculatiors (yr) & $2.000 E+03$ & $1.000 E+06$ \\
\hline$R 012 j$ & Initial principal radionuclide $(p C i / g): A c-227$ & $2.300 \Sigma+01$ & $0.000 E+00$ \\
\hline $\mathbf{R} 012$ & Initial principal radionuclide $(p c i / g): P a-231$ & $2.300 E+01$ & $0.000 E+00$ \\
\hline R012 & Initial principal radionuclide $(p \mathrm{ci} / \mathrm{g})$ : $\mathrm{Pb}-210$ & $4.992 E+02$ & $0.000 E+00$ \\
\hline R012 & Initial principal radionuclide ( $\mathrm{pCi} / \mathrm{g})$ : Ra-226 & $4.992 E+02$ & $0.000 E+00$ \\
\hline 2012 & latitial principal radionuclide $(p c i / 9)$ : Th-230 & 4.994E+02 & $0.000 E+00$ \\
\hline R012 & Initial principsl radionuclide (pCi/g): U-234 & $4.994 E+02$ & $0.000 E+00$ \\
\hline R012 & Initial principal radionuclide $(p c i / g): \quad U-235$ & $2.300 E+01$ & $0 . J 00 E+00$ \\
\hline R012 & Initial principal radionuclide (pCi/g): U-238 & 4.994E+02 & $0.000 E+00$ \\
\hline R013 & Cover depth $(m)$ & $0.000 E+00$ & $0.000 E+90$ \\
\hline R013 & Density of contaminated zone $(g / \mathrm{cm}+3)$ & $1.600 E+00$ & 1.600 \\
\hline $\mathbf{R} 013$ & Contaminated zone erasion rate $(m / y r)$ & $0.000 E+00$ & $1.000 E-03$ \\
\hline$n 013$ & Contaminated zone total porosity & 4.920E-01 & 4.000E-01 \\
\hline R013 & Contaminated zone effective porosity & $1.300 E-01$ & 2.00 \\
\hline R013 & Contaminated zone hydraulic conductivity $(\mathrm{m} / \mathrm{yr})$ & $3.260 E+01$ & $1.000 \mathrm{E}+01$ \\
\hline R013 & Contaminated zone b parameter & $1.040 E+01$ & $5.300 E+00$ \\
\hline R013 & Evapotranspiration coefficient & $6.000 E-0 i$ & \\
\hline R013 & Precipitation (m/yr) & $9.600 \mathrm{E}-01$ & $1.000 E+00$ \\
\hline R013 & Irrigation $(m / y r)$ & $2.000 E-01$ & $2.000 E-01$ \\
\hline R013 & Irrigation mode & overhead & \\
\hline R013 & Runoff coefficient & $2.000 \mathrm{E}-01$ & $2.000 \mathrm{E}-01$ \\
\hline R013 & Watershed area for nearby stream or pond $\left(m^{*+2}\right)$ & $1.000 E+06$ & $1.000 E+06$ \\
\hline $\mathbf{R 0 1 4}$ & Density of saturated zone $\left(9 / \mathrm{cm}^{* *} 3\right)$ & $1.600 \mathrm{E}+00$ & $1.600 E+00$ \\
\hline R014 & Saturated zone total porosity & $4.000 E-01$ & $4.000 E-01$ \\
\hline $\mathrm{R} 014$ & Saturated zone effective porosity & $2.000 E-01$ & $2.000 E-01$ \\
\hline R014 & Saturated zone hydraulic conductivity $(\mathrm{m} / \mathrm{yr})$ & $1.000 E+02$ & $1.000 E+02$ \\
\hline R014 & Saturated zone hydraul ic gradient & | $2.000 E-02$ & $2.000 E \cdot 02$ \\
\hline R014 & Distance from surface to water table (m) & $1.520 E+00$ & $5.000 E+00$ \\
\hline R014 & Water table drop rate $(\mathrm{m} / \mathrm{yr})$ & $0.000 E+00$ & $1.000 E-03$ \\
\hline R014 & Hell pum intake depth ( $m$ below water table) & | $1.000 E+01$ & $1.000 E+01$ \\
\hline R014 & Model: Mondispersion (MD) or Mass-Balance (MB) & I ND & IND \\
\hline R015 & Number of unsaturated zone strata & 1 & 11 \\
\hline
\end{tabular}


Site-Specific Parameter Sumary (cont inued)

\begin{tabular}{|c|c|c|c|}
\hline Menu $\mid$ & Parameter & Used & Default \\
\hline R015 & Unsat. zone 1 , thickness (m) & $1.220 E+00$ & $4.000 \mathrm{E}+00$ \\
\hline R015 I & Unsat. zone 1, soil density $\left(0 / \mathrm{cm}^{+*} 3\right)$ & $1.600 E+00$ & $1.600 \mathrm{E}+00$ \\
\hline R015 i & Unsat. zone 1, total porosity & $4.920 E-01$ & $4.000 E-01$ \\
\hline 2015 & Unsat. zone 1, effective porosity & $1.300 E-01$ & 2.000E-01 \\
\hline RO15 i & Unsat. zone 1, soil-specific b paraneter & $1.040 E+01$ & $5.300 E+00$ \\
\hline R015 & Unsat. zone 1, hydraulic conductivity ( $\mathrm{m} / \mathrm{yr})$ & $\mid 3.260 E+01$ & $1.000 E+02$ \\
\hline R016 i & Distribution coefficients for $1 c-227$ & & \\
\hline 2016 | & Contaninated zone $(\mathrm{cm}+1+3 / g)$ & $2.000 E+01$ & $2.000 E+01$ \\
\hline R016 | & Unsaturated zone $1(\mathrm{~cm} * 3 / \mathrm{g})$ & $2.000 E+01$ & $2.000 E+01$ \\
\hline R016 I & Saturated zone $(\mathrm{cm}+3 / g)$ & $2.000 E+09$ & $2.000 E+01$ \\
\hline $\begin{array}{r}\text { R016 I } \\
\text { I }\end{array}$ & Leach rate $(/ \gamma r)$ & $0.000 E+00$ & $0.000 E+00$ \\
\hline R016 | & Distribution coefficients for $\mathrm{Pa}-231$ & & \\
\hline R016 & Contaninated zone $(\mathrm{cm}+3 / \mathrm{s})$ & $5.000 E+01$ & $5.000 E+01$ \\
\hline 2016 & Ursaturated zone $1(\mathrm{~cm}+\mathrm{e} / \mathrm{g})$ & $5.000 E+01$ & $5.000 E+01$ \\
\hline R016 | & Saturated zone $(c+4+4 / g)$ & | $5.000 E+01$ & $5.000 E+01$ \\
\hline R016 | & Leach rate $(/ y r)$ & $\left.\right|^{0.000 E+00}$ & $0.000 E+00$ \\
\hline R016 | & Distribution coefficients for $\mathrm{Pb}-210$ & & \\
\hline $\mathbf{R 0 1 6}$ & 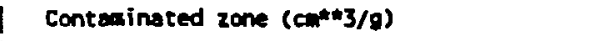 & $1.000 E+02$ & $1.000 E+02$ \\
\hline R016 & Unseturated zone $1(\mathrm{~cm}+3 / \mathrm{g})$ & $1.000 E+02$ & $1.000 E+02$ \\
\hline R016 & Saturated zone $\left(\mathrm{cm}^{*} \mathrm{k} / \mathrm{g} / \mathrm{g}\right)$ & $1.000 E+02$ & $1.000 \mathrm{E}+02$ \\
\hline R016 & Leach rate $(/ y r)$ & $0.000 E+00$ & $0.000 E+00$ \\
\hline R016 | & Distribution coefficients for Ra-226 & & \\
\hline R016 | & Contaminated zone $\left(\mathrm{cm}^{*} * 3 / \mathrm{g}\right)$ & $7.000 E+01$ & $7.000 E+01$ \\
\hline R016 | & Unsaturated zone $1\left(\mathrm{can}^{k+3 / g}\right)$ & | 7.000E+01 & $7.000 E+01$ \\
\hline R016 | & Saturated zone $\left(\mathrm{cm}^{2}+3 / \mathrm{g}\right)$ & | $7.000 E+01$ & $7.000 E+01$ \\
\hline R016 & Leach rate (/yr) & $\left.\right|^{0.000 E+00}$ & $0.000 E+00$ \\
\hline R016 & Distribution coefficients for th- 230 & 1 & \\
\hline R016 & Contaminated zone $(c-m+3 / g)$ & $6.000 E+04$ & $6.000 E+04$ \\
\hline R016 & Unsaturated zone $1(c-m * 3 / g)$ & $6.000 E+04$ & $6.000 E+04$ \\
\hline R016 | & Soturated zone $(\mathrm{cm} * 3 / \mathrm{s})$ & $6.000 E+04$ & $6.000 E+04$ \\
\hline R016 & Leach rate $(/ y r)$ & $0.000 E+00$ & $0.000 E+00$ \\
\hline R016 i & Distribution coefficients for U-234 & & \\
\hline R016 | & Contasinated zone $(\cos * 3 / g)$ & $5.000 E+01$ & $5.000 E+01$ \\
\hline $\mathbf{R} 016$ | & Unsaturated 2 one $1(\mathrm{~cm}+3 / g)$ & $5.000 E+01$ & 5.000E+01 \\
\hline R016 | & Saturated $z$ one $(\mathrm{cm}+\mathrm{H} / \mathrm{g})$ & $5.000 E+01$ & $5.000 E+01$ \\
\hline R016 | & Leach rate $(/ y r)$ & $1^{0.000 E+00}$ & $0.000 E+00$ \\
\hline R016 । & Distribution coefficients for U-235 & & \\
\hline R016 & Contaninated zone $\left(\mathrm{cm}^{4 * 3 / 9}\right)$ & 5.000E*01 & $5.000 E+01$ \\
\hline R016 & Unsaturated zone $1\left(\mathrm{~cm}^{+43 / g)}\right.$ & $5.000 E+01$ & $5.000 E+01$ \\
\hline R016 & Saturated $20 n e\left(\mathrm{~cm}^{+}+3 / \mathrm{g}\right)$ & 15.000E+01 & $5.000 E+01$ \\
\hline $\mathbf{R 0 1 6} \mid$ & Leach rate $(/ y r)$ & $0.000 E+00$ & $0.000 E+00$ \\
\hline
\end{tabular}


Site-Specific Paraneter Sumary (cont inued)

\begin{tabular}{|c|c|c|c|}
\hline Menu | & Paranister & Used & Default \\
\hline R016 & Distribution coefficients for U-239 & & \\
\hline R016 I & Contaminated zone $\left(\mathrm{cm}^{* * 13 / g)}\right.$ & $5.000 E+01$ & $5.000 E+01$ \\
\hline RO16 i & Unsaturated zone $1\left(\mathrm{~cm}^{++3 / 9}\right)$ & $5.000 E+01$ & $5.000 E+01$ \\
\hline RO16 | & Saturated zone $(\mathrm{cm} \| 3 / \mathrm{g})$ & $5.000 E+01$ & $5.000 E+01$ \\
\hline R016 & Leach rate $(/ y r)$ & $0.000 E+00$ & $0.000 E+00$ \\
\hline K017 & Inhalation rate $\left(m^{+m} 3 / y r\right)$ & $8.400 E+03$ & $8.400 E+03$ \\
\hline R017 & Mass loading for inhalation $\left(g / m^{* * 3}\right)$ & $2.000 E-04$ & $2.000 E-04$ \\
\hline R017 & Dilution length for airborne dust, inhalation $(m)$ | & $3.000 E+00$ & $3.000 E+00$ \\
\hline R017 & occupancy factor, inhalation & $4.500 E-01$ & $4.500 E=01$ \\
\hline R017 & Dccupancy and shielding factor, external gamma & $5.600 E-01$ & $6.000 E-01$ \\
\hline R017 & Shape factor, external gamma & $1.000 E+00$ & $1.000 \mathrm{E}+00$ \\
\hline R018 & Fruits, vegetables and grain consumption $(\mathrm{kg} / \mathrm{\gamma r})$ | & $1.600 E+02$ & $1.600 E+02$ \\
\hline R018 & Leafy vegetable consumption (kg/yr) & $? .400 E+01$ & $1.400 \mathrm{E}+01$ \\
\hline R018 & Milk consumption (L/Yr) & $0.000 E+00$ & $9.200 E+01$ \\
\hline R018 & Meat and poul try consumption (kg/yr) & $0.000 E+00$ & $6.300 E+01$ \\
\hline R018 & Fish consumption $(\mathrm{kg} / \mathrm{yr})$ & $0.000 E+00$ & $5.400 E+00$ \\
\hline R018 & Other seafood consumption (kg/yr) & $0.000 E+00$ & $9.000 E-01$ \\
\hline R018 & Drinking water intake (L/Yr) & $0.000 E+00$ & 4.100E+02 \\
\hline R018 & Fraction of drinking water from site & $0.000 E+00$ & $1.000 E+00$ \\
\hline R018 & Fraction of aquatic food from site & $0.000 E+00$ & $5.000 E-01$ \\
\hline R019 i & | Livestock fodder intake for meat (kg/day) & $6.800 E+01$ & 6.800E+01 \\
\hline R019 & Livestock fodder intake for milk (kg/day) & $5.500 E+01$ & $5.500 E+01$ \\
\hline R019 & Livestock water intake for weai (L/day) & $5.080 E+01$ & $5.000 E+01$ \\
\hline R019 & I Livestcck water intake for milk (L/day) & $1.600 E+02$ & $1 .: 00 E+02$ \\
\hline R019 & Mass loading for foliar deposition $\left(g / \mathrm{m}^{+*}+3\right)$ & $1.000 E-06$ & $1.000 \mathrm{E}-04$ \\
\hline R019 & Depth of scil mixing layer (m) & $1.500 E-01$ & $1.500 E-01$ \\
\hline R019 & Depth of roots $(m)$ & $9.000 \mathrm{E}-01$ & $9.000 \mathrm{E}-01$ \\
\hline R019 & Orinking water fraction from ground water & $1.000 E+00$ & $1.000 E+00$ \\
\hline R019 | & | Livestock water fraction from ground water & $1.000 E+00$ & $1.000 E+00$ \\
\hline R019 । & I Irrigation fraction from ground water & $1.000 E+00$ & $1.000 E+00$ \\
\hline
\end{tabular}




\section{B.2 REFERENCES}

Ecology and Environment, Inc., 1980, Field Investigations of Uncontrolled Hazardous Waste Sites; A Preliminary Assessment of New Jersey Zinc Co., Palmerton, PA, TDD No. F3-8007-28, EPA No. PA-280, prepared for Environmental Protection Agency.

Gilbert, T.L., et al., 1989, A Manual for Implementing Residual Radioactive Material Guidelines, ANL/ES-160, DOE/CH/8901, prepared by Argonne National Laboratory for the U.S. Department of Energy, June.

Griffin, J., 1990, personal communication from J. Griffin (Horsehead Resource Development, Palmerton, Pa., Superintendent of Environmental Affairs) to M. Nimmagadda (Argonne National Laboratory), June 28.

U.S. Department of Health, Education, and Welfare, 1970, Radiological Health Handbook, Bureau of Radiological Health, Rockville, Md.

Williams, W.A., 1990, "Specific Comments on the Draft Preliminary Dose Assessment for the Palmerton Ore Storage Site, Palmerton, Pennsylvania," letter from W.A. Williams (Department of Energy, Division of Eastern Area Programs, Office of Environmental Restoration) to C. Yu (Argonne National Laboratory), Aug. 17. 


\section{APPENDIX C:}

\section{RESRAD RESULTS}

The RESRAD summary results for Scenarios A, B, C, and D are presented on the following pages. 
Conteleinated Zane Diansions

$\begin{aligned} \text { Area: } & 3.00 \text { mawe meters } \\ \text { Thicknass: } & 0.30 \text { mers } \\ \text { Cover Depth: } & 0.00 \text { meters }\end{aligned}$

Initial Soil Concentrations, $p c i / 0$

\begin{tabular}{ll}
\hline Ac -227 & $2.300 E+01$ \\
$P a-231$ & $2.300 E+01$ \\
$P b-210$ & $6.902 E+02$ \\
$R=-226$ & $4.992 E+02$ \\
$T h-230$ & $6.994 E+02$ \\
$U-234$ & $4.994 E+02$ \\
$U-235$ & $2.300 E+01$ \\
$U-238$ & $4.994 E+02$
\end{tabular}

Total DOEe TOOSE(t), mrealyr

Busic Rediation Dose Limit = $100 \mathrm{mrea} / \mathrm{yr}$

Iotal Mixture sum $A(t)$ - Fraction of Insic Dose Lielt Received at Tine ( $t$ )

\begin{tabular}{|c|c|c|c|c|c|c|c|c|c|c|}
\hline (yeara): & 0 & 1 & 10 & 100 & 200 & 400 & 600 & 800 & 1000 & 2000 \\
\hline & $1.006 E+\infty 0$ & $9.550 E-01$ & $9.0325-01$ & 6.674E-01 & $2.709 E-01$ & $1.850 E-01$ & $1.729-01$ & $1.706 E-01$ & $1.697 \mathrm{E}-01$ & $1.664 E-01$ \\
\hline & $1.006 E-02$ & $9.950 E \cdot 03$ & $9.032 z-03$ & 4.474E-03 & $2.7095-03$ & $1.850 \mathrm{E}-03$ & $1.729 E-03$ & $1.706 \mathrm{E}-03$ & $1.697 \mathrm{E}-03$ & $1.664 E-03$ \\
\hline
\end{tabular}

Maxien roose(t): $1.006 E+00$ erealyr at $t=0$ years

Total Dose Contributions TDCSE $(i, p, t)$ for Individual Radionuclides (i) and Pathways ( $p$ ), erea/yr

$$
\text { At } t=0 \text { years }
$$

Water Independent Pathury

\begin{tabular}{|c|c|c|c|c|c|}
\hline el ide & Grand & Dust & Plent & Mest & Milk \\
\hline$=-227$ & $6.600 x-03$ & $1.621 E-01$ & $0.000 E+00$ & $0.000 E+\infty 0$ & $0.000 E+00$ \\
\hline-231 & $3.680 E-06$ & $2.758 E-02$ & $0.000 E+00$ & $0.000 E+00$ & $0.000 E+00$ \\
\hline$b-210$ & $1.596 E-04$ & $9.670 \mathrm{D}-03$ & $0.000 E+00$ & $0.000 E+00$ & $0.000 E+00$ \\
\hline $2-226$ & $5.670 E-01$ & $3.630 E \cdot 03$ & $0.000 E+00$ & $0.000 E+00$ & $0.000 E+00$ \\
\hline Th-230 & 7.087E-05 & $1.676 E-01$ & $0.000 E+\infty 0$ & $0.000 E+\infty 0$ & $0,000 E+00$ \\
\hline$U \cdot 234$ & $6.917 E-05$ & $5.900 x-02$ & $0.000 E+00$ & $0.000 E+00$ & $0.000 E+\infty 0$ \\
\hline U-235 & $1.507 \mathrm{E}-03$ & $2.546 E-03$ & $0.000 E+00$ & $0.000 E+\infty 0$ & $0.000 E+00$ \\
\hline 4-238 & $6.561 E-03$ & $5.526-02$ & $0.000 E+\infty 0$ & $0.000 E+00$ & $0.000 E+00$ \\
\hline & & 6.461E-01 & $0.000 E+\infty 0$ & $0.000 E+00$ & 0.0 \\
\hline
\end{tabular}

Water Dependent Pathways

\begin{tabular}{|c|c|c|c|c|c|}
\hline Uoter & Fish & $P \operatorname{lan} t$ & Meat & Milk & Total \\
\hline $0.000 E++0$ & $0.000 E+00$ & $0.000 E+00$ & $0.000 E+00$ & $0.000 E+00$ & $1.467 \mathrm{t}-01$ \\
\hline $0.000 E+00$ & $0.000 E+00$ & $0.000 E+00$ & $0.000 E+00$ & $0.000 E+00$ & 2.79 \\
\hline $0.000 E+00$ & $0.000 E+100$ & $0.000 E * 00$ & $0.000 E+00$ & $0.000 E+00$ & 9.829 \\
\hline $0.000 E+00$ & $0,000 E \bullet 00$ & $0.000 E+00$ & $0.000 E+00$ & $0.000 E+00$ & 5.50 \\
\hline $0.000 E+00$ & $0.000 E+00$ & $0.000 E+00$ & $0.000 E+00$ & $0.000 E+00$ & $1.675 E-01$ \\
\hline $0.000 E+00$ & $0.000 E+00$ & $0.000 E+00$ & $0.000 E+00$ & $0.000 E+00$ & $5.993 E-02$ \\
\hline $0.000 E+00$ & $0.000 E+00$ & $0.000 E+00$ & $0.000 E+00$ & $0.000 E+00$ & $4.0535-03$ \\
\hline $0.000 E+00$ & $0.000 E+00$ & $0.000 E+00$ & $0.000 E+00$ & $0.000 E+00$ & $5.904 E-02$ \\
\hline $0.000 E+00$ & $0.000 E+00$ & $0.000 E+00$ & $0.000 E+00$ & $0.000 E+00$ & $1.006 E+00$ \\
\hline
\end{tabular}

Total Dose Contributions toose $(i, p, t)$ far Individusl Redionuclides (i) and Pathways ( $p$ ), percent

$$
\text { at } t=0 \text { yeart }
$$

\begin{tabular}{|c|c|c|c|c|c|}
\hline el lide & Grand & Dust & Plent & Meat & Milk \\
\hline$=-227$ & $6.570 E-01$ & $1.612 E+01$ & $0.000 E+\infty 0$ & $0.000 E+\infty 0$ & $0.000 E+00$ \\
\hline$\cdot .231$ & $3.656 E-C 2$ & $2.740 E-00$ & $0.020=+00$ & $0.000 E+00$ & $0.000 E+00$ \\
\hline$b-210$ & $1.586 E-02$ & $9.607 \mathrm{E}-01$ & $0.000 E+00$ & $0.000 E+00$ & $0.000 E+00$ \\
\hline$=-226$ & $5.635 E+01$ & $3.614 E-01$ & $0.000 E+00$ & $0.000 E+00$ & $0.000 E+00$ \\
\hline Th-230 & $7.061 E-03$ & $1.465 E+01$ & $0.000 E+\infty 0$ & $0.000 E+00$ & $0.000 E+00$ \\
\hline$u-234$ & $6 . \cos E-03$ & $5.950 E+00$ & $0.000 \mathrm{E}+00$ & $0.000 E+00$ & $0.000 E+00$ \\
\hline 235 & $1.6900-01$ & $2.5296-01$ & $0.000 E+00$ & $0.000 E+\infty 0$ & $0.000 E+00$ \\
\hline $4-238$ & $4.531 E=01$ & $5.4922 E+00$ & $0.000 E+00$ & $0.000 E+00$ & $0.000 E+00$ \\
\hline & &. $.45 \mathrm{ca}$ & $0.000 E+\infty 0$ & $0.000 E+00$ & $0.000 t=00$ \\
\hline
\end{tabular}

Water Independent Pathways

\begin{tabular}{|c|c|c|c|c|c|}
\hline Uater & fish & Plant & Meat & Milk & Totel \\
\hline $0.000 E+00$ & $0.000 E+00$ & $0.000 E+00$ & $0.000 E+00$ & $0.000 E+00$ & $1.458 E+01$ \\
\hline $0.000 \mathrm{E}+00$ & $0.000 E+00$ & $0.000 E+00$ & $0.000 E+00$ & $0.000 E+00$ & $2.777 \mathrm{E}+00$ \\
\hline $0.000 E+00$ & $0.000 E+00$ & $0.000 E+00$ & $0.000 E+00$ & $0.000 E+00$ & $9.7656-01$ \\
\hline $0.000 E+00$ & $0.000 E+00$ & $0.000 E+00$ & $0.000 E+00$ & $0.000 E \cdot 00$ & $5.471 E+01$ \\
\hline $0.000 E+00$ & $0.000 E+00$ & $0.000 E+00$ & $0.000 E+00$ & $0.000 E \cdot 00$ & $1.465 E+01$ \\
\hline $0.000 E+00$ & $0.000 E+00$ & $0.000 E+00$ & $0.000 E+00$ & $0.000 E+00$ & $5.955 E+00$ \\
\hline $0.000 E+00$ & $0.000 E+00$ & $0.000 E+00$ & $0.000 E+00$ & $0.000 E+00$ & $6.027 \mathrm{E}-01$ \\
\hline $0.000 E+00$ & $0.000 E+00$ & $0.000 E+00$ & $0.000 E+00$ & $0.000 E+00$ & $5.845 E+00$ \\
\hline $0.000 E+00$ & $0.000 E+00$ & $0.000 E+00$ & $0.000 E+00$ & $0.000 E+00$ & $1.000 E+02$ \\
\hline
\end{tabular}

Water Dependent Pathways 
Contaminated Zone Dimensions

$\begin{array}{rc}\text { Area: } & 9662.00 \text { square meters } \\ \text { ihickness: } & 1.00 \text { meters } \\ \text { Cover Depth: } & 0.15 \text { meters }\end{array}$

Initial Soil Cancentrations, $\mathrm{pCi} / \mathrm{g}$

$\begin{array}{ll}\text { Ac-227 } & 1.900 E-01 \\ \text { Pa-231 } & 1.900 E-01 \\ \text { Pb-210 } & 2.530 E+00 \\ \text { Ra-226 } & 2.530 E+00 \\ \text { Th-230 } & 4.200 E+00 \\ U-234 & 4.200 E+00 \\ U-235 & 1.900 E-01 \\ U-238 & 4.200 E+00\end{array}$

Total Dose TDOSE(t), mrem/yr

Basic Radiation Dose Limit = $100 \mathrm{mren} / \mathrm{yr}$ Total Mixture Sun M(t) = fraction of Basic Dose Linit Received at $T$ ime (t)

\begin{tabular}{|c|c|c|c|c|c|c|c|c|c|c|}
\hline t (years): & 0 & 1 & 10 & 100 & 200 & 400 & 600 & 800 & 1000 & 2000 \\
\hline TOOSE $(t):$ & 2.317E-01 & $2.311 E-01$ & $2.258 E-01$ & 1.817E-01 & $1.458 E-01$ & $1.006 E-01$ & $7.681 E-02$ & $6.418 E-02$ & 5.745E-02 & 4.974E-02 \\
\hline$M(t):$ & $2.31 \pi=-03$ & 2.311E-03 & $2.258 E-03$ & $1.81 \pi-03$ & $1.458 E-03$ & $1.006 E-03$ & $7.681 E-04$ & $6.418 E-04$ & $5.745 E-04$ & 4.974E-04 \\
\hline
\end{tabular}

Maxinum Toose(t): 2.317E-01 area/yr at $t=0$ years

Total Oose Contributions ToOse(i,p,t) for Individual Radionucl ides (i) and Pathways ( $p$ ), mrem/yr

$$
\text { At } t=0 \text { years }
$$

Ueter Independent Pathways

\begin{tabular}{|c|c|c|c|c|c|}
\hline Nuclide & Ground & Dust & Plant & Meat & Mitk \\
\hline$A c-227$ & $2.534 E-03$ & $0.000 E+00$ & $0.000 E+00$ & $0.000 E+00$ & $0.000 E+00$ \\
\hline$P a-231$ & $6.174 E-05$ & $0.000 E \div 90$ & $0.000 E+00$ & $0.000 E+00$ & $0.000 E+00$ \\
\hline $\mathrm{Pb}-210$ & $1.183 E-05$ & $0.000 E+00$ & $0.000 E+00$ & $0.000 E+00$ & $0.000 E+00$ \\
\hline$R a-226$ & $2.216 E-01$ & $0.000 E+00$ & $0.000 E+00$ & $0.000 E+00$ & $0.000 E+00$ \\
\hline Th-230 & 8.695E-06 & $0.000 E+00$ & $0.000 E+00$ & $0.000 E+00$ & $0.000 E+00$ \\
\hline $\mathbf{U} \cdot \mathbf{2 3 4}$ & $6.035 E-06$ & $0.000 E+00$ & $0.000 E+00$ & $0.000 E+00$ & $0.000 E+00$ \\
\hline$U-235$ & $1.818 E-06$ & $0.000 E+00$ & $0.000 E+00$ & $0.000 E+00$ & $0.800 E+00$ \\
\hline$U-238$ & $7.285 E-03$ & $0.000 E+00$ & $0.000 E+00$ & $0.000 E+00$ & $0.000 E+00$ \\
\hline To & $2.31 \pi-01$ & $0.000 E+00$ & $0.000 E+00$ & $0.000 E+00$ & $0.000 E+00$ \\
\hline
\end{tabular}

Water Oependent Pathways

\begin{tabular}{|c|c|c|c|c|c|}
\hline Water & Fish & Plant & Meat & Milk & Total \\
\hline $0.000 E+00$ & $0.000 E+00$ & $0.000 E+00$ & $0.000 E+00$ & $0.000 E+00$ & $2.534 E-03$ \\
\hline $0.000 E+00$ & $0.000 E+00$ & $0.000 E+00$ & $0.000 E+00$ & $0.000 E+00$ & $6.174 E-05$ \\
\hline $0.000 E+00$ & $0.000 E+00$ & $0.000 E+00$ & $0.000 E+50$ & $0.000 E+00$ & $1.183 E-05$ \\
\hline $0.000 E+00$ & $0.000 E+00$ & $0.000 E+00$ & $0.000 E+00$ & $0.000 E+00$ & $2.216 E-01$ \\
\hline $0.000 E+00$ & $0.000 E+00$ & $0.000 E+00$ & $0.000 E+00$ & $0.000 E+00$ & $8.695 E-06$ \\
\hline $0.000 E+00$ & $0.000 E+00$ & $0.000 E+00$ & $0.000 E+00$ & $0.000 E+00$ & $6.035 E-06$ \\
\hline $0.000 E+00$ & $0.000 E+00$ & $0.000 E+00$ & $0.000 E+00$ & $0.000 E+00$ & $1.818 E-04$ \\
\hline $0.000 E+00$ & $0.000 E+00$ & $0.000 E+00$ & $0.000 E+00$ & $0.000 E+00$ & $7.265 E-03$ \\
\hline $0.000 E+00$ & $0.000 E+00$ & $0.000 E+00$ & $0.000 E+00$ & $0.000 E+00$ & 2.317E-01 \\
\hline
\end{tabular}

Total Dose Contributions IDOSE( $i, p, t)$ for Individual Radionuclides (i) and Pathways ( $p$ ), percent

$$
\text { At } t=0 \text { vears }
$$

\begin{tabular}{|c|c|c|c|c|c|}
\hline Nucl ide & Ground & Dust & Plant & Meat & Milk \\
\hline$A c-227$ & $1.094 \mathrm{E}+00$ & $0.000 E+00$ & $0.000 E+00$ & $0.000 E+00$ & $0.000 E+00$ \\
\hline$P a-231$ & $2.665 E-02$ & $0.000 E+00$ & $0.000 E+00$ & $0.000 E+00$ & $0.000 E+00$ \\
\hline$P b-210$ & $5.107 \varepsilon-03$ & $0.000 E+00$ & $0.000 E+00$ & $0.000 E+00$ & $0.000 E+00$ \\
\hline$R A-226$ & $9.564 E+01$ & $0.000 E+00$ & $0.000 \varepsilon+00$ & $0.000 E+00$ & $0.000 E+00$ \\
\hline Th-230 & $3.753 E-03$ & $0.000 E+00$ & $0.000 E+00$ & $0.000 E+00$ & $0.000 E+00$ \\
\hline$U-234$ & $2.605 E-03$ & $0.000 E+00$ & $0.000 E+00$ & $0.000 E+00$ & $0.000 E+00$ \\
\hline$u-235$ & $7.847 \mathrm{E}-02$ & $0.000 E+00$ & $0.000 E+00$ & $0.000 E+00$ & $0.000 E+00$ \\
\hline$U-238$ & $3.145 E+00$ & $0.000 E+00$ & $0.000 E+00$ & $0.000 E+00$ & $0.000 E+00$ \\
\hline Total & & $0.000 E+00$ & $0.00,0 E+00$ & $0.000 E+00$ & $0.000 E+00$ \\
\hline
\end{tabular}

Water Indopendent Pathways

\begin{tabular}{|c|c|c|c|c|c|}
\hline Uater & Fish & Plant & Meat & Milk & Total \\
\hline $0.000 E+00$ & $0.000 E+00$ & $0.000 E+00$ & $0.000 E+00$ & $0.000 E+00$ & $1.094 E+00$ \\
\hline $0.000 E+00$ & $0.000 E+00$ & $0.000 E+00$ & $0.000 E+00$ & $0.000 E+00$ & $2.665 \mathrm{E}-02$ \\
\hline $0.000 E+00$ & $0.000 E+00$ & $0.000 E+00$ & $0.000 E+00$ & $0.000 E+00$ & $5.107 E-03$ \\
\hline $0.000 E+00$ & $0.000 E+00$ & $0.000 E+00$ & $0.000 E+00$ & $0.000 E+00$ & $9.564 E+01$ \\
\hline $0.000 E+00$ & $0.000 E+00$ & $0.000 E+00$ & $0.000 E+00$ & $0.000 E+00$ & $3.753 E-03$ \\
\hline $0.000 E+00$ & $0.000 E+00$ & $0.000 E+00$ & $0.000 E+00$ & $0.000 E+00$ & $2.605 E-03$ \\
\hline $0.000 E+00$ & $0.000 E+00$ & $0.000 E+00$ & $0.000 E+00$ & $0.000 E+00$ & $7.84 \pi-02$ \\
\hline $0.000 E+00$ & $0.000 E+00$ & $0.000 E+00$ & $0.000 E+00$ & $0.000 E+00$ & $3.145 E+00$ \\
\hline $0,000 E+00$ & $0.000 E+00$ & $0.000 E+00$ & $0.000 E+00$ & $0.000 E+00$ & $1.000 E+02$ \\
\hline
\end{tabular}

Water Dependent Pathways 
Conteminuted Zone Dimenetons

$\begin{aligned} \text { Ares: } & 9662.00 \text { equre meters } \\ \text { Thieknote: } & 1.00 \text { enters } \\ \text { Cover Depth: } & 0.15 \text { enters }\end{aligned}$

Initial soil concentration $\mathrm{PCl} / \mathrm{g}$

$\begin{array}{ll}\text { AC- } 227 & 1.900 E-01 \\ P u-231 & 1.900 E-01 \\ P b-210 & 2.530 E+00 \\ \text { Ra-226 } & 2.530 E+00 \\ \text { Ih-230 } & 4.200 E+00 \\ U-236 & 4.200 E+00 \\ U-235 & 1.900 E-01 \\ U .236 & 4.200 E+00\end{array}$

Totsl Dow Those(t), arearr

Devic Radiation Dose Lialt = 100 moreyr

\begin{tabular}{|c|c|c|c|c|c|c|c|c|c|c|}
\hline$t$ (yesrs): & 0 & 1 & 10 & 100 & 200 & 400 & 600 & 800 & 1000 & 2000 \\
\hline TDorse $(t)$ & $6.61 \%-01$ & 6.602E-01 & $6.45 Z Z-01$ & 5.192E-01 & $4.164 E-01$ & $2.876 E-01$ & $2.185 E-01$ & $1.834 \mathrm{E}-01$ & $1.641 E-01$ & $1.421 E-01$ \\
\hline$m(\varepsilon)$ & $6.619 E-03$ & 6.60ZE-03 & 6.652 E -03 & $5.192 E-03$ & $4.164 E-03$ & $2.876 E-03$ & $2.195 \mathrm{E}-03$ & $1.834 E \cdot 03$ & $1.641 E-03$ & $1.421 E-03$ \\
\hline
\end{tabular}

Maxien roose $(t)$ : $6.6195-01$ armyr ot $t=0$ yeare

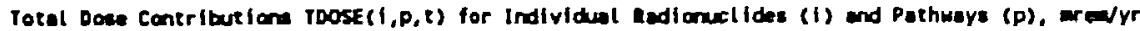

At 0 years

Whter Indepondent Pathwart

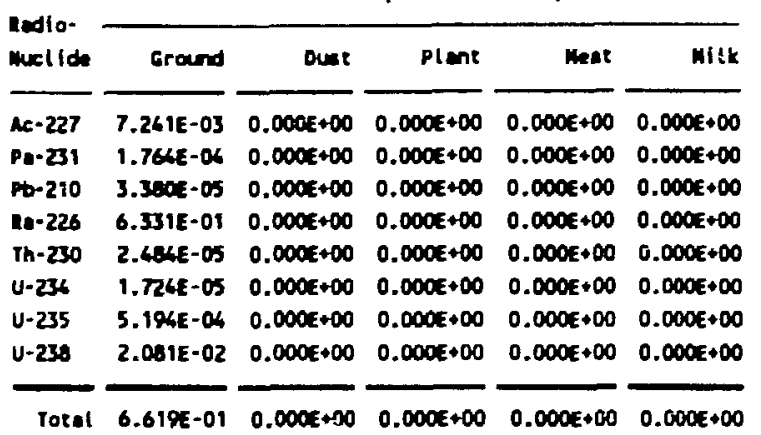

Wotor Dependent Pathuars

\begin{tabular}{|c|c|c|c|c|c|}
\hline Heter & Ffth & $P \operatorname{lan} t$ & mest & Mitu & Total \\
\hline $0.000 E+00$ & $0.000=+\infty 0$ & $0.000 E+00$ & $0.000=+00$ & $0.000 E \$ 00$ & $7.241 E-03$ \\
\hline $0.000 E+00$ & $0.000 \varepsilon+00$ & $0.000 E+00$ & $0.000 E+00$ & $0.000 E+00$ & $1.764 E-06$ \\
\hline $0.000 E+00$ & $0.0005+00$ & $0.000 \varepsilon+00$ & $0.0005+00$ & $0.000[+00$ & $3.300=-05$ \\
\hline $0.000 E+\infty 0$ & $0.000 \varepsilon+00$ & $0.000 \leqslant+00$ & $0.000 \varepsilon+00$ & $0.000 \varepsilon+00$ & $6.331 E \cdot 01$ \\
\hline $0.0005+00$ & $0.000 E+00$ & $0.000 E+00$ & $0.000 \mathrm{E}+\infty$ & $0.000 E+00$ & $2.684 E-05$ \\
\hline $0.000 \varepsilon+\infty 0$ & $0.000 E+00$ & $0.000<\bullet 00$ & $0.000 E+00$ & $0.000 E+00$ & $1.726 E-05$ \\
\hline $0.000 E+00$ & $0.000 E+00$ & $0.000 \varepsilon+00$ & $0.000 E+00$ & $0.000 E+00$ & 5.194E-04 \\
\hline $0.000 E+00$ & $0.000 E+00$ & $0.000 E+00$ & $0.000 E+00$ & $0.000 \varepsilon+00$ & 2.0S4E-02 \\
\hline $0.000 E+\infty 0$ & $0.000 \varepsilon+00$ & $0.000<+00$ & $0.000 E \nrightarrow 00$ & $0.000 E+00$ & $6.618-01$ \\
\hline
\end{tabular}

Total Dose Contribution Toose $(i, p, t)$ for Individul Redioncel ides (i) and pothweys (p), percent

$$
\text { At } 0 \text { years }
$$

Uater Independent Pethway

\begin{tabular}{|c|c|c|c|c|c|}
\hline Mucl ide & Ground & Duse & Plent & Weat & Milk \\
\hline$A c-227$ & $1.0 \% 4 E+00$ & $0.000 E+00$ & $0.000 E+00$ & $0.000 E+\infty 0$ & $0.000 E+00$ \\
\hline$P=-231$ & $2.665 E-02$ & $0.000 E+00$ & $0.000 E+\infty 0$ & $0.000 E+00$ & $0.000 E+00$ \\
\hline$p b-210$ & 5.107E-03 & $0.000 E * 00$ & $0.000 E \bullet 00$ & $0.000 E+00$ & $0.000 E \div 00$ \\
\hline $1=-226$ & $9.564 E+01$ & $0.000 E+00$ & $0.0006+00$ & $0.000 E+\infty 0$ & $0.000 E \bullet 00$ \\
\hline$T h-230$ & $3.753 E-03$ & $0.000 E+00$ & $0.000=+00$ & $0.000 E+00$ & $0.000 E+00$ \\
\hline$v \cdot 236$ & $2.605 E-03$ & $0.004=000$ & $0.000=400$ & $0.000 \leq \bullet 00$ & $0.000 E \bullet 00$ \\
\hline$U \cdot 235$ & $7.84 \pi-02$ & $0.000=00$ & $9.000=000$ & $0.000 E+00$ & $0,000 E \div 00$ \\
\hline$u \cdot 230$ & $3.145 E+00$ & $0.000 E+00$ & $0.000=000$ & $0.000 E+\infty 0$ & $0.000 E+\infty 0$ \\
\hline & & $0.000=-00$ & $0.000 E+00$ & $0.000 E+00$ & $0.000 E+00$ \\
\hline
\end{tabular}

Water Dependent Pathways

\begin{tabular}{|c|c|c|c|c|c|}
\hline Water & Fich & Plant & Meat & Milk & Total \\
\hline $0.000 E+\infty 0$ & $0.000 \mathrm{E} \cdot 00$ & $0.000 E+00$ & $0.000 E * 00$ & $0.000 E+C O$ & $1.094 E+00$ \\
\hline $0.000 E+00$ & $0.000 E+00$ & $0.000 E=00$ & $0.000 \epsilon-00$ & $0.000 E+00$ & $2.665 \mathrm{E} \cdot 02$ \\
\hline $0.000<+00$ & $0.000 E+00$ & $0.000 E+00$ & $0.000 €+00$ & $0.000<+00$ & 5. 10TE-03 \\
\hline $0.000 E+00$ & $0.000 E \bullet 00$ & $0.000 E \bullet 00$ & $0.000 E \bullet 00$ & $0.000 E+00$ & $9.564 E \bullet 01$ \\
\hline $0.000<+00$ & $0.000 E+00$ & $0.000 E+00$ & $0.000 € \div 00$ & $0.000 E \bullet 00$ & $3.753 E-03$ \\
\hline $0.000 E+00$ & $0.000 E \div 00$ & $0.000 E+00$ & $0.000 E * 00$ & $0.000 E \div 00$ & $2.605 E \cdot 05$ \\
\hline $0.000 E+\infty 0$ & 0.0005 .30 & $0.000 E+00$ & $0.000<<00$ & $0.000 E+00$ & $7.84 \pi-02$ \\
\hline $0.000 E+\infty 0$ & $0.000 \varepsilon+00$ & $0.000=\bullet 00$ & $0.000 E+00$ & $0.000 E \bullet 00$ & $3.165 E \bullet 00$ \\
\hline $0.000 E \bullet 00$ & $0.000 E * 00$ & $0.000 E \bullet 00$ & $0.000 E * 00$ & $0.000 E * 00$ & $1.000 E+02$ \\
\hline
\end{tabular}


Sumary : Scenario D

Contaminated zone Dinensions

$\begin{aligned} \text { Area: } & \mathbf{3 . 0 0} \text { square eners } \\ \text { Thickness: } & 0.30 \text { meters } \\ \text { Cover Depth: } & 0.00 \text { meters }\end{aligned}$

File: SD.DAT

Initial Soil Concentrations, pci/o

$\begin{array}{ll}A c-227 & 2.300 E+01 \\ P a-231 & 2.300 E+01 \\ P b-210 & 4.992 E+02 \\ R a-226 & 4.992 E+02 \\ \text { Th-230 } & 4.994 E+02 \\ U-234 & 4.994 E+02 \\ U-235 & 2.300 E+01 \\ U-238 & 4.994 E+02\end{array}$

Total Dose Toose(t), arem/yr

Basic Radiation Dose Limit = $100 \mathrm{mren} / \mathrm{yr}$

Total Mixture Sum $M(t)=$ Fraction of Basic Dose Limit Received at Time $(t)$

\begin{tabular}{|c|c|c|c|c|c|c|c|c|c|c|}
\hline (years): & 0 & 1 & 10 & 100 & 200 & 400 & 600 & 800 & 1000 & 2000 \\
\hline TDOSE (t): & $3.630 E+02$ & $3.581 E+02$ & $3.186 E+02$ & $1.345 E+02$ & $7.655 E+01$ & $5.423 E+01$ & $5.203 E+01$ & $5.161 E+01$ & $5.135 E+01$ & $5.021 E+01$ \\
\hline$M(t)$ & $3.630 E+00$ & $3.581 E+00$ & $3.186 E+00$ & $1.345 E+00$ & $7.655 \mathrm{E}-01$ & $5.423 E-01$ & $5.203 E-01$ & 5.161E-01 & 5. 135E-01 & 5.021E-01 \\
\hline
\end{tabular}

Maximm TOOSE(t): $3.630 E+02 \mathrm{mreay} / \mathrm{r}$ at $t=0$ years

Total Dose Coneributions TDOSE(i,p,t) for Individual Rodionuclides (i) and Pathways (p), aren/yr

$$
\text { At } t=0 \text { years }
$$

\begin{tabular}{|c|c|c|c|c|c|}
\hline \multirow{2}{*}{$\begin{array}{l}\text { Radio- } \\
\text { Nuclió }\end{array}$} & \multicolumn{3}{|c|}{ Hater Independent Pathways } & & \\
\hline & Ground & Dust & Plant & Heat & Hilk \\
\hline$A c-227$ & $1.720 E+00$ & $4.264 E+01$ & $7.527 \mathrm{E}-02$ & $0.000 E+00$ & $0.000 E+00$ \\
\hline Pa-231 & $1.374 E-01$ & B. $274 E+00$ & 5.520E-02 & $0.000 E+00$ & $0.000 E+00$ \\
\hline Pb-210 & $5.960 E-02$ & 2. S01E +00 & $1.9796+01$ & $0.000 E+00$ & $0.000 E+00$ \\
\hline $\operatorname{Ra}-226$ & $2.042 E+02$ & $1.001 E+00$ & $6.726 E-02$ & $0.000 E+00$ & $0.000 E+00$ \\
\hline Th- 230 & $2.646 E-02$ & $4.422 E+01$ & $9.689-02$ & $0.000 E+00$ & $0.000 E+00$ \\
\hline $\mathrm{U}-\mathbf{2 3 6}$ & $1.836 E-02$ & $1.796 E+01$ & $2.853 E-02$ & $0.000 E+00$ & $0.000 E+00$ \\
\hline$u \cdot 235$ & $5.627 E-01$ & $7.637 \mathrm{E}-01$ & $1.255 E-03$ & $0.000 E+00$ & $0.000 E+00$ \\
\hline U-238 & $1.703 E+00$ & $1.658 E+01$ & 2.T24E-02 & 0. COOE +00 & $0.000 E+00$ \\
\hline Total & $2.095 E+02$ & $1.344 E+02$ & $2.014 E+01$ & $0.000 E+00$ & $0.000 E+00$ \\
\hline
\end{tabular}

\begin{tabular}{|c|c|c|c|c|c|}
\hline Uater & Fish & Plant & Heat & Milk & Totel \\
\hline $0.000 E+00$ & $0.000 E+00$ & $0.000 E+00$ & $0.000 E+00$ & $0.000 E+00$ & $4.444 E+01$ \\
\hline $0.000 E+00$ & $0.000 E+00$ & $0.000 E+00$ & $0.000 E+00$ & $0.000 E+00$ & $8.466 E+00$ \\
\hline 0.000E+00 & 0.000E+00 & $0.00 c \tau+00$ & $0.000 E+00$ & $0.000 E+00$ & $2.275 E+01$ \\
\hline $0.000 E+00$ & $0.000 E+00$ & $0.000 E+00$ & $0.000 E+00$ & $0.000 E+00$ & $2.054 E+02$ \\
\hline $0.000 E+00$ & $0.000 E+00$ & $0.000 E+00$ & $0.000 E+00$ & $0.000 E+00$ & $4.434 E+01$ \\
\hline $0.000 E+00$ & $0.000 E+00$ & $0.000 E+00$ & $0.000 E+00$ & $0.000 E+00$ & $1.801 E+01$ \\
\hline $0.000 E+00$ & $0.000 E+00$ & $0.000 E+00$ & $0.000 E+00$ & $0.000 E+00$ & $1.328 \varepsilon+00$ \\
\hline $0.000 E+00$ & $0.000 E+00$ & $0.000 E+00$ & $0.000 E+00$ & $0.000 E \& 00$ & $1.831 E+01$ \\
\hline $0.000 E+00$ & $0.000 E+00$ & $0.000 E+00$ & $0.000 E+00$ & $0.000 E+00$ & $3.630 \varepsilon+02$ \\
\hline
\end{tabular}

Total Dose Contributions TDOSE $(i, p, t)$ for Individual Redionuelides (i) and Pathways (p), percent

$$
\text { At } t=0 \text { years }
$$

\begin{tabular}{|c|c|c|c|c|c|}
\hline Nuel ide & Ground & Dust & Plant & Meat & Milk \\
\hline$A c-227$ & 4.738E-01 & $1.175 E+01$ & 2.07SE-02 & $0.000 E+00$ & $0.000 E+00$ \\
\hline $\mathrm{Pa}-231$ & $3.784 \mathrm{E}-02$ & $2.279 E+00$ & $1.520 E-02$ & $0.000 E+00$ & $0.000 E+00$ \\
\hline$P b-210$ & $1.642 E-02$ & $7.991 E-01$ & $5.451 E+00$ & $0.000 E+00$ & $0.000 E+00$ \\
\hline$R a-226$ & $5.626 E+01$ & 3.006E-01 & $1.853 E-02$ & $0.000 E+00$ & $0.000 E+00$ \\
\hline Th-230 & $7.288 E-03$ & $1.218 E+01$ & $2.669 E-02$ & $0.000 E+00$ & $0.000 E+00$ \\
\hline$U-234$ & $5.057 \mathrm{E}-03$ & $6.969 \varepsilon+00$ & $7.003 E-03$ & $0.000 E+00$ & $0.000 E+D 0$ \\
\hline$U-235$ & $1.550 E-01$ & $2.104 E-01$ & $3.456 E-06$ & $0.000 E+00$ & $0.000 E^{-00}$ \\
\hline $\mathrm{U}-238$ & $4.690 E-01$ & $4.568 E+00$ & $7.503 E-03$ & $0 . \operatorname{COOE}+00$ & $0.000 E+00$ \\
\hline Total & $5.742 E+01$ & 3.703E+01 & $5.548 E+00$ & $0.000 E+00$ & U. UOUE +UO \\
\hline
\end{tabular}

Uater Independent Pathways
Water Dependent Pathways

\begin{tabular}{cccccccc}
\hline Water & \multicolumn{2}{c}{ Fish } & Plant & Meat & Milk & Total \\
\cline { 3 - 6 } & & & & & & \\
$0.000 E+00$ & $0.000 E+00$ & $0.000 E+00$ & $0.000 E+00$ & $0.000 E+00$ & $1.224 E+01$ \\
$0.000 E+00$ & $0.000 E+00$ & $0.000 E+00$ & $0.000 E+00$ & $0.000 E+00$ & $2.332 E+00$ \\
$0.000 E+00$ & $0.000 E+00$ & $0.000 E+00$ & $0.000 E+00$ & $0.000 E+00$ & $6.266 E+00$ \\
$0.000 E+00$ & $0.000 E+00$ & $0.000 E+00$ & $0.000 E+00$ & $0.000 E+00$ & $5.657 E+01$ \\
$0.000 E+00$ & $0.000 E+00$ & $0.000 E+00$ & $0.000 E+00$ & $0.000 E+00$ & $1.221 E+01$ \\
$0.000 E+00$ & $0.000 E+00$ & $0.000 E+00$ & $0.000 E+00$ & $0.000 E+00$ & $4.961 E+00$ \\
$0.000 E+00$ & $0.000 E+00$ & $0.000 E+00$ & $0.000 E+00$ & $0.000 E+00$ & $3.657 E-01$ \\
$0.000 E+00$ & $0.000 E+00$ & $0.000 E+00$ & $0.000 E+00$ & $0.000 E+00$ & $5.044 E+00$ \\
\hline $0.000 E+00$ & $0.000 E+00$ & $0.000 E+00$ & $0.000 E+00$ & $0.000 E+00$ & $1.000 E+02$
\end{tabular}

